
\title{
25 Research Square \\ Effect of Dark Septate Endophytes on Plant Performance of Artemisia Ordosica and Associated Soil Microbial Community Under Salt Stress
}

Lifeng Hou

Hebei University

Xia Li

Hebei University

Xueli He ( $\nabla$ xlh3615@126.com )

Hebei University College of Life Sciences

Yiling Zuo

Hebei University

Dongdong Zhang

Hebei University

Lili Zhao

Hebei University

Research article

Keywords: Dark septate endophytes, Artemisia ordosica, plant performance, soil microbial community composition, $\mathrm{NaCl}$ stress, symbiosis

Posted Date: November 24th, 2020

DOl: https://doi.org/10.21203/rs.3.rs-107827/v1

License: (c) (i) This work is licensed under a Creative Commons Attribution 4.0 International License.

Read Full License 


\section{Abstract}

Background: Fungal endophytes can improve plant tolerance to abiotic stress, however, the role of these plant-fungal interactions in desert species ecology and their management implications remain unclear. This study aimed to assess whether dark septate endophytes (DSE) can shift the performance of Artemisia ordosica and associated soil microbial community under salt stress.

Methods: We investigated the effects of three DSE (Alternaria chlamydosporigena [AC], Paraphoma chrysanthemicola $[\mathrm{PC}]$ and Bipolaris sorokiniana $[\mathrm{BS}]$ ) isolated from desert habitats on plant morphology, physiology and rhizosphere soil microhabitat of Artemisia ordosica seedlings under different $\mathrm{NaCl}$ concentrations $(0 \%, 0.1 \%, 0.2 \%$, and $0.3 \%)$ in a growth chamber.

Results: Three DSE strains could colonize the roots of $A$. ordosica, and the symbiotic response with host plants depended on DSE species and $\mathrm{NaCl}$ concentration. The greatest benefits associated with DSE occurred under $0.1 \% \mathrm{NaCl}$. Specifically, AC improved root morphology, and increased total biomass and superoxide dismutase (SOD) activity; PC increased root morphology, root biomass, and glutathione (GSH) and indoleacetic acid (IAA) contents; and BS promoted SOD activity and GSH and IAA contents. DSE reduced the root $\mathrm{Na}^{+}$content. Interestingly, BS promoted gram-positive $(\mathrm{G}+)$ and gram-negative (G -) bacteria under $0.1 \% \mathrm{NaCl}$ and the abundance of $\mathrm{AM}$ fungi under $0.2 \%$ and $0.3 \% \mathrm{NaCl}$. PC positively affected fungi, AM fungi, $\mathrm{G}$ - bacteria and actinomycetes under $0.2 \%$ and $0.3 \% \mathrm{NaCl}$, while $\mathrm{AC}$ increased the abundance of all examined microbes under $0.3 \% \mathrm{NaCl}$. A structural equation modeling (SEM) demonstrated that DSE not only positively affects $A$. ordosica performance but also directly or indirectly impacts soil microbes by regulating the soil organic carbon (SOC), available phosphorus (AP), and alkaline nitrogen (AN) content.

Conclusions: DSE isolated from A. ordosica enhanced the root development of host plants and altered the soil nutrient content and soil microbiota under different $\mathrm{NaCl}$ concentrations, possibly contributing to plant growth and ecological adaptability under saline environment. These results contribute to the understanding of the ecological function of DSE in desert ecosystems and may be used to promote vegetation restoration in salinized desert areas.

\section{Background}

Soil degradation is a severe environmental problem worldwide, especially in arid and semiarid regions. The evaporation in these regions greatly exceeds the precipitation, and salt dissolved in the groundwater may accumulate at the soil surface through capillary movement [1]. In addition to water stress, salt accumulation is becoming a severe current and future problem faced by plants [2,3]. Salt stress normally causes ion toxicity, osmotic shock and oxidative stress, which disrupt plant metabolic processes and hinder plant growth [4-6]. Thus, plants have evolved a series of physiological and biochemical mechanisms to alleviate these negative effects [7-9]. Soil microbes largely inhabit plants in their natural habitat, and some soil microbes are stress tolerant and capable of stimulating plant growth [10]. Plants 
not only provide important habitats for their associated microbiome but also provide photosynthates for microbial growth [11, 12]. Moreover, the soil-associated microbiome has an important function in ecological functions and nutrient availability, further affecting plant growth and productivity $[13,14]$. Previous studies have shown that plant tolerance to stress, such as drought, salinity and high temperature, is closely related to the colonization of endophytic fungi $[15,16]$. Hence, the resource excavation of these associated endophytic fungi containing dark septate endophytes (DSE) is vital for improving the health and productivity of plants, especially for plant growth and vegetation restoration in degraded soils $[17,18]$.

DSE represent a diverse group of endophytic fungi mainly involving ascomycetes, which propagate via conidia or asexual reproduction. These fungi inhabit the root tissues of healthy plants, and the dark septal hyphae and microsclerotia grown intracellularly and intercellularly are their main characteristics [19-21]. DSE have been shown to harbor an extensive range of hosts and have a broad ecological distribution; they are typically found in plants growing in stressful habitats, including those experiencing drought, salt, or heavy metal stress [22-28]. Previous studies have shown that host plant responses to DSE are variable, including positive, neutral and negative responses [29]. Indeed, DSE can alter plant growth, alter the accumulation of auxin and other chemical components, and prevent the development of abiotic resistance in host plants [30-32]. However, the effects of DSE on host plant performance and fitness remain unknown, especially under salt-stress conditions.

DSE isolated from wetlands or coastal areas have been found to alleviate the adverse effects of salt stress on plant growth [33-36]. Pan et al. [33] reported that Curvularia spp. isolated from Suaeda salsa could alleviate the adverse effects of salt stress on Populus tomentosa by increasing both antioxidant enzyme activity and the content of chlorophyll and proline in the leaves. Farias et al. [34] reported that inoculation with DSE improved the absorption of nitrogen and phosphorus in Vigna unguiculata under salt stress, which was beneficial for increasing plant growth and net photosynthesis. In addition, rhizosphere-associated microbes have important functions in the maintenance of soil nutrient availability, which have positive impacts on the tolerance of host plants to salt stress [37,38]. Similar results have also been reported in other stressful environments. He et al. [15] reported that DSE improved the root development and antioxidant enzyme activity of Glycyrrhiza uralensis and altered the soil organic nutrition content and microbiota composition, which may also promote plant growth and survival under water deficit conditions. To date, the effects of DSE on the rhizospheric microbial community of host plants under salt stress have not been studied.

Artemisia ordosica (Asteraceae) is one of the dominant sand-fixing shrub species that is widely distributed in the arid and semiarid regions of northwestern China and is an important medicinal and feed plant species. This species is tolerant to drought, soil infertility, wind erosion and sand burial and has been widely used for phytoremediation as a wind break and to sand fix, maintaining biodiversity and ecosystem stability in northwestern China $[39,40]$. Excessive amounts of $\mathrm{Na}^{+}$in the soil have been found to be a major cause of plant damage and restrict vegetation restoration in arid and semiarid regions [1, 41]. Investigating the response of host plants and soil microbial communities to DSE under salt-stress 
conditions is highly important to improve the bioremediation of saline soils in arid and semiarid regions. In this study, we conjecture that DSE fungi could provide more beneficial effects on promoting the growth of $A$. ordosica and altering the soil microbial community and physicochemical properties in the rhizosphere of $A$. ordosica under conditions of salt stress. Based on our conjecture, we researched the response of (1) the growth of plants, (2) the antioxidant system, (3) $\mathrm{Na}^{+}$and $\mathrm{K}^{+}$ions, (4) soil physical and chemical properties, and (5) the composition of soil microbes to DSE inoculation under conditions of salt stress. We expect that the results will reveal the impact mechanism of DSE inoculation resistance to salinization conditions on the growth and production of plants and further explore the potential of these fungi to enhance plant stress tolerance and symbiotic function during vegetation restoration in saline desert regions.

\section{Results}

\section{DSE colonization analysis}

After harvest, typical DSE structures including dark septate hyphae and microsclerotia in inoculated plants were observed (Fig. 1). The total colonization rate was $10 \%-60 \%$ (Fig. 2). The colonization rate of Paraphoma chrysanthemicola (PC) peaked under $0.1 \% \mathrm{NaCl}$. The maximum colonization rate of Bipolaris sorokiniana (BS) occurred under $0.3 \% \mathrm{NaCl}$, and no significant differences were found among the different $\mathrm{NaCl}$ treatments. However, the total colonization by Alternaria chlamydosporigena (AC) in the $A$. ordosica roots significantly decreased with increasing $\mathrm{NaCl}$ concentration.

\section{Plant biomass production}

The shoot, root and total biomass and root:shoot ratio of host plants were significantly influenced by the interactions between DSE species and $\mathrm{NaCl}$ stress treatment (Table 1). Inoculation with AC resulted in an increase in the shoot biomass and total biomass under $0.1 \% \mathrm{NaCl}$ compared to the control plants. No significant differences in shoot and total biomass were found in the other treatments (Fig. 3a, c). 
Table 1

Two-way analysis of variance of the effects of dark septate endophytes (DSE) inoculation and $\mathrm{NaCl}$ stress on the growth and morphology parameters of Artemisia ordosica.

\begin{tabular}{|c|c|c|c|c|c|c|c|c|c|}
\hline & DSE & & & $\mathrm{NaCl}$ stre & & & DSE $\times N$ & Cl stres & \\
\hline & $F$ & $P$ & $n_{p}^{2}$ & $F$ & $P$ & $\eta_{p}^{2}$ & $F$ & $P$ & $n_{p}^{2}$ \\
\hline $\begin{array}{l}\text { Shoot } \\
\text { biomass }\end{array}$ & 3.17 & 0.038 & 0.229 & 81.621 & $\hat{0} .001$ & 0.884 & 2.75 & 0.017 & 0.436 \\
\hline Root biomass & 25.43 & $<.001$ & 0.704 & 150.596 & $\begin{array}{l}<.001 \\
0.01\end{array}$ & 0.934 & 4.758 & $<.001$ & 0.572 \\
\hline $\begin{array}{l}\text { Total } \\
\text { biomass }\end{array}$ & 3.739 & 0.021 & 0.26 & 108.139 & 0.001 & 0.91 & 2.724 & 0.018 & 0.434 \\
\hline $\begin{array}{l}\text { Root:shoot } \\
\text { ratio }\end{array}$ & 17.465 & <. 001 & 0.621 & 7.155 & 0.001 & 0.401 & 3.049 & 0.009 & 0.462 \\
\hline Plant height & 11.449 & <. 001 & 0.518 & 71.54 & <. 001 & 0.87 & 1.404 & 0.228 & 0.283 \\
\hline $\begin{array}{l}\text { Shoot } \\
\text { branching }\end{array}$ & 12.082 & $\begin{array}{l}<.001 \\
\text { L }\end{array}$ & 0.531 & 109.254 & $\stackrel{<}{0.001}$ & 0.911 & 6.533 & $\hat{0} .001$ & 0.648 \\
\hline Root length & 63.019 & <. 001 & 0.855 & 298.463 & <. 001 & 0.965 & 10.594 & 0.001 & 0.749 \\
\hline $\begin{array}{l}\text { Root surface } \\
\text { area }\end{array}$ & 50.486 & $\langle .001$ & 0.826 & 276.307 & $\hat{0} .001$ & 0.963 & 11.165 & $<.001$ & 0.758 \\
\hline Root diameter & 1.02 & 0.397 & 0.087 & 4.089 & 0.014 & 0.277 & 0.477 & 0.879 & 0.118 \\
\hline Root volume & 15.764 & $\hat{0.001}$ & 0.596 & 95.558 & $\begin{array}{l}<.001 \\
0\end{array}$ & 0.9 & 5.313 & < 0.001 & 0.599 \\
\hline
\end{tabular}

DSE increased the root biomass under $0 \% \mathrm{NaCl}$ compared with the control plants. With increasing $\mathrm{NaCl}$ concentration, compared with that of the control plants, only inoculation with PC increased the root biomass under $0.1 \%$ and $0.3 \% \mathrm{NaCl}$, whereas $\mathrm{BS}$ inoculation decreased the root biomass under $0.2 \%$ and $0.3 \% \mathrm{NaCl}$ (Fig. 3b).

The root:shoot ratio was significantly enhanced by DSE under $0 \% \mathrm{NaCl}$ compared to control plants. With increasing $\mathrm{NaCl}$ concentration, only inoculation with $\mathrm{PC}$ increased the root:shoot ratio under $0.3 \% \mathrm{NaCl}$. However, inoculation with $\mathrm{AC}$ and BS decreased the root:shoot ratio under $0.1 \% \mathrm{NaCl}$ (Fig. 3d).

\section{Plant morphological traits}


DSE species and $\mathrm{NaCl}$ stress treatment significantly influenced plant height. Furthermore, the interaction between DSE species and $\mathrm{NaCl}$ stress treatment significantly affected shoot branching (Table 1). Inoculation with AC positively affected plant height and was significant under $0.2 \%$ and $0.3 \% \mathrm{NaCl}$ (Fig. 4a). A significant increase in shoot branching occurred only under $0 \% \mathrm{NaCl}$. However, with increasing $\mathrm{NaCl}$ concentration, inoculation with $\mathrm{AC}$ reduced shoot branching compared with that of the control plants under $0.1 \%$ and $0.3 \% \mathrm{NaCl}$ (Fig. 4b).

Interactions between DSE species and $\mathrm{NaCl}$ stress treatment significantly influenced the root length, root volume and root surface area of $A$. ordosica (Table 1). Inoculation with DSE promoted the root growth of A. ordosica under $0 \% \mathrm{NaCl}$. In addition, under $0.1 \% \mathrm{NaCl}, \mathrm{AC}$ and $\mathrm{PC}$ inoculation enhanced the root length, root volume and root surface area compared to control plants. As the $\mathrm{NaCl}$ concentration increased, only inoculation with PC significantly enhanced the root length, root volume and root surface area under $0.3 \%$ $\mathrm{NaCl}$. Furthermore, no significant differences in root diameter were found under any treatment (Fig. 4c, d, e, f).

\section{Antioxidant enzyme activities in the leaves}

Interactions between DSE species and $\mathrm{NaCl}$ stress treatment on the superoxide dismutase (SOD) activity and glutathione (GSH) content in host plants were significant (Table 2). DSE inoculation resulted in increased SOD activity and GSH content of the leaves under $\mathrm{NaCl}$ stress. AC and BS inoculation increased SOD activity under $\mathrm{NaCl}$ stress compared with that of the control plants, while PC inoculation improved SOD activity only under $0.2 \% \mathrm{NaCl}$ (Fig. 5a). BS inoculation increased the GSH content compared to the control plants under $\mathrm{NaCl}$ stress, $\mathrm{PC}$ inoculation increased the $\mathrm{GSH}$ content only under $0.1 \% \mathrm{NaCl}$, and $\mathrm{AC}$ inoculation increased the $\mathrm{GSH}$ content under $0.2 \%$ and $0.3 \% \mathrm{NaCl}$ (Fig. $5 \mathrm{~b}$ ). 
Table 2

Two-way analysis of variance of the effects of dark septate endophytes (DSE) inoculation and $\mathrm{NaCl}$ stress on the physiological parameters of Artemisia ordosica.

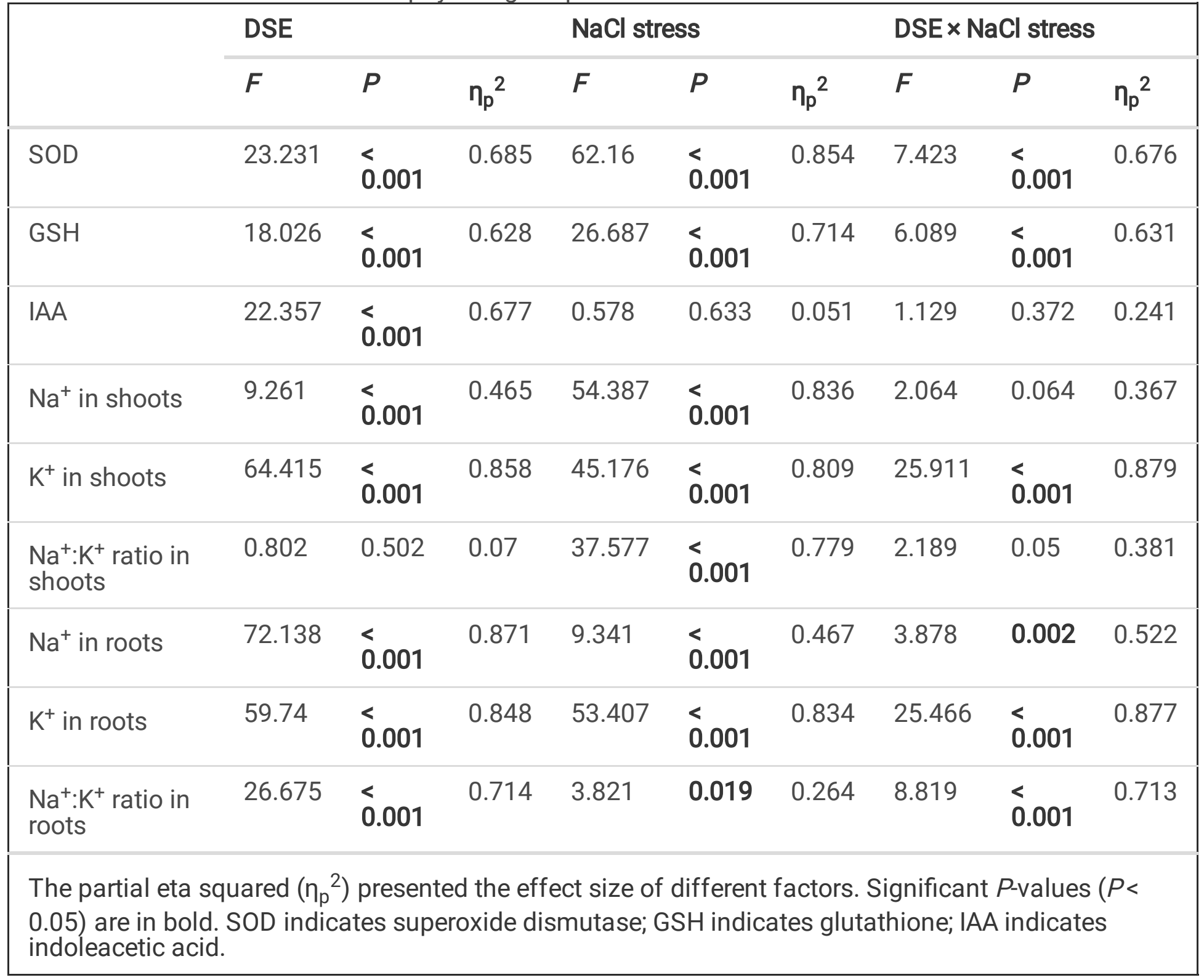

\section{Indoleacetic acid (IAA) content in the roots}

DSE species significantly influenced IAA production in A. ordosica roots regardless of the $\mathrm{NaCl}$ stress treatment (Table 2). Inoculation with PC and BS increased the IAA content compared to the control plants under all $\mathrm{NaCl}$ treatments. Moreover, $\mathrm{AC}$ inoculation had a positive effect on IAA content under $0.2 \%$ and $0.3 \% \mathrm{NaCl}$, but there were no significant differences (Fig. 5c).

\section{$\mathrm{Na}^{+}$and $\mathrm{K}^{+}$content}

DSE species and $\mathrm{NaCl}$ stress treatment had significant interaction effects on the $\mathrm{K}^{+}$content in the shoots of $A$. ordosica. Moreover, the $\mathrm{Na}^{+}$content in the shoots was remarkably influenced by DSE species and $\mathrm{NaCl}$ stress treatment (Table 2). BS inoculation increased the $\mathrm{Na}^{+}$and $\mathrm{K}^{+}$content under $\mathrm{NaCl}$ stress 
compared with that in the control plants. No significant difference in the $\mathrm{Na}^{+}: \mathrm{K}^{+}$ratio was found under $\mathrm{NaCl}$ stress treatment (Fig. 6a, c, e).

DSE species and $\mathrm{NaCl}$ stress treatment had significant interaction effects on the $\mathrm{Na}^{+}$and $\mathrm{K}^{+}$content and the $\mathrm{Na}^{+}: \mathrm{K}^{+}$ratio in the roots of $A$. ordosica (Table 2). Compared to the controls, the $\mathrm{Na}^{+}$content in roots of DSE-inoculated plants decreased under $\mathrm{NaCl}$ stress treatments. Inoculation with $\mathrm{AC}$ increased the $\mathrm{K}^{+}$ content in the roots under $0.2 \%$ and $0.3 \% \mathrm{NaCl}$ compared to control plants. Furthermore, inoculation with $\mathrm{AC}$ and $\mathrm{PC}$ decreased the $\mathrm{Na}^{+}: \mathrm{K}^{+}$ratio under $0.2 \%$ and $0.3 \% \mathrm{NaCl}$ (Fig. $6 \mathrm{~b}, \mathrm{~d}, \mathrm{f}$ ).

\section{Soil physicochemical properties}

Interactions between DSE species and $\mathrm{NaCl}$ stress treatment remarkably influenced the soil organic carbon (SOC) content, while the soil available phosphorus (AP) content was significantly affected by DSE species and $\mathrm{NaCl}$ stress treatment. Moreover, the soil alkaline nitrogen (AN) content was significantly affected only by DSE species (Table 3). PC inoculation reduced the SOC content under all $\mathrm{NaCl}$ treatments and reduced the AP content under $0.1 \% \mathrm{NaCl}$ compared with that in the control plants. AC inoculation reduced the SOC content only under $0.3 \% \mathrm{NaCl}$. However, $\mathrm{BS}$ inoculation reduced the content of SOC and AP under both $0.1 \%$ and $0.3 \% \mathrm{NaCl}$ and increased the $\mathrm{AN}$ content under both $0 \%$ and $0.3 \%$ $\mathrm{NaCl}$ (Fig. 7). 
Table 3

Two-way analysis of variance of the effects of dark septate endophytes (DSE) inoculation and $\mathrm{NaCl}$ stress on soil factors and microbial composition in rhizosphere of Artemisia ordosica.

\begin{tabular}{|c|c|c|c|c|c|c|c|c|c|}
\hline & \multicolumn{3}{|l|}{ DSE } & \multicolumn{3}{|c|}{$\mathrm{NaCl}$ stress } & \multicolumn{3}{|c|}{ DSE $\times \mathrm{NaCl}$ stress } \\
\hline & $F$ & $P$ & $\eta_{p}^{2}$ & $F$ & $P$ & $\eta_{p}^{2}$ & $F$ & $P$ & $\eta_{p}^{2}$ \\
\hline SOC & 22.29 & $\begin{array}{l}<.001 \\
\text { L }\end{array}$ & 0.676 & 12.885 & $\dot{0} 001$ & 0.547 & 4.376 & 0.001 & 0.552 \\
\hline AN & 10.552 & ¿ 0.001 & 0.497 & 0.754 & 0.528 & 0.066 & 0.357 & 0.947 & 0.091 \\
\hline AP & 10.126 & $\hat{0}_{0.001}$ & 0.487 & 37.508 & $\hat{0} .001$ & 0.779 & 0.82 & 0.602 & 0.187 \\
\hline Fungi & 5.18 & 0.005 & 0.327 & 1.501 & 0.233 & 0.123 & 2.896 & 0.013 & 0.449 \\
\hline AM Fungi & 4.525 & 0.009 & 0.298 & 2.62 & 0.068 & 0.197 & 3.48 & 0.004 & 0.495 \\
\hline G - bacteria & 5.024 & 0.006 & 0.32 & 1.381 & 0.266 & 0.115 & 5.076 & $\langle .001$ & 0.588 \\
\hline $\mathrm{G}+$ bacteria & 10.511 & $\begin{array}{l}<.001 \\
0\end{array}$ & 0.496 & 2.756 & 0.058 & 0.205 & 7.577 & $\hat{0} .001$ & 0.681 \\
\hline Actinomycetes & 3.099 & 0.04 & 0.225 & 1.934 & 0.144 & 0.154 & 3.976 & 0.002 & 0.528 \\
\hline \multicolumn{10}{|c|}{$\begin{array}{l}\text { The partial eta squared }\left(\eta_{p}{ }^{2}\right) \text { presented the effect size of different factors. Significant } P \text {-values }(P< \\
0.05) \text { are in bold. SOC indicates soil organic carbon; AN indicates alkaline nitrogen; AP indicates } \\
\text { available phosphorus; } G \text { - bacteria indicates gram-negative bacteria; } G+\text { bacteria indicates gram- } \\
\text { positive bacteria. }\end{array}$} \\
\hline
\end{tabular}

\section{Soil microbial community composition}

Interactions between DSE species and $\mathrm{NaCl}$ stress treatment significantly affected the microbial community composition in the rhizospheric soil of $A$. ordosica (Table 3). Under $0.1 \% \mathrm{NaCl}$, BS inoculation enhanced gram-negative ( $(-)$ and gram-positive $(G+)$ bacterial abundance compared with those of the control plants. Under $0.2 \% \mathrm{NaCl}, \mathrm{PC}$ inoculation increased the abundance of $\mathrm{G}$ - bacteria, actinomycetes, fungi and arbuscular mycorrhizal (AM) fungi compared to controls, whereas BS inoculation only enhanced the abundance of $\mathrm{AM}$ fungi. When the $\mathrm{NaCl}$ concentration reached $0.3 \%$, compared with the control, the plants inoculated with AC presented an increased abundance of soil microbes; the plants inoculated with PC presented an increased abundance of $\mathrm{G}$ - bacteria, actinomycetes, fungi and AM fungi, whereas BS inoculated plants presented an enhanced abundance of fungi and AM fungi (Fig. 8).

\section{Correlation analysis}

Spearman's rank correlation test indicated that significant relationships existed among DSE species, $\mathrm{NaCl}$ stress, soil factors, soil microbial composition, and the growth parameters of $A$. ordosica (Table S1). On 
the basis of the correlation coefficients (R-value), structural equation modeling (SEM) further revealed the relative effects of DSE species, $\mathrm{NaCl}$ stress and soil factors on the growth of $A$. ordosica and on the rhizospheric microbial composition $\left(X^{2}=78.527, \mathrm{df}=60, P=0.055, \mathrm{CFI}=0.969, \mathrm{TLI}=0.953\right.$, and RMESA $=$ $0.081 ;$ Fig. 9). The SEM indicated that DSE directly and significantly positively influenced the plant hormone (IAA) content and antioxidant enzyme (SOD) activity and directly negatively affected the root $\mathrm{Na}^{+}$content. Moreover, DSE significantly indirectly affected plant hormone (IAA) content, antioxidant enzyme (SOD) activity, and root growth (root length and biomass) by affecting soil factors (SOC, AP, and AN). In addition, DSE directly and significantly positively influenced the abundance of $G+$ bacteria and fungi, whereas DSE had significant indirect effects on the abundance of $G$ - bacteria by affecting soil factors (SOC). In addition, $\mathrm{G}-$ bacteria and root biomass directly negatively affected the $\mathrm{Na}^{+}$content in the roots.

\section{Discussion}

The current study reported for the first time the effects of DSE isolated from an arid desert environment on the growth of $A$. ordosica under $\mathrm{NaCl}$ stress. In all inoculated plants, typical DSE structures (dark septate hyphae and microsclerotia) in roots were observed, revealing that these DSE can be regarded as effective colonizers of the roots of $A$. ordosica under all $\mathrm{NaCl}$ treatments. DSE hyphae are important media for water transport and nutrient exchange between plants and DSE, while microsclerotia are also considered to be propagules or hypopus, which constitute important ecological strategies to plants for tolerating environmental stress [23, 42]. However, different DSE strains showed variable colonization patterns with increasing $\mathrm{NaCl}$ concentrations, and similar results of studies of DSE improving the heavy metal tolerance of plants have been reported [43-45]. The primary reason may be that the stress affected the growth and physiological metabolism of the DSE hyphae $[46,47]$, thus regulating the infection ability of the DSE on plant roots. In addition, the growth response of $A$. ordosica to DSE colonization was also strain dependent, which is consistent with previous findings [29,46]. Specifically, AC resulted in a significant increase in $A$. ordosica biomass accumulation under $0.1 \% \mathrm{NaCl}$, while PC exerted beneficial effects under both $0.1 \%$ and $0.3 \% \mathrm{NaCl}$. However, BS inoculation decreased the root biomass under $0.2 \%$ and $0.3 \% \mathrm{NaCl}$. Therefore, the variable effects of different DSE on plants may be related to the different colonization rates and DSE species, which need further investigation.

The root system is the primary plant part that senses salt stress, and roots can respond rapidly through changes in elongation [48] and function [49]. In the present study, DSE promoted the growth of the root system under $\mathrm{NaCl}$ stress, although the effects depended on the DSE species. Under the low-stress treatment $(0.1 \% \mathrm{NaCl}), \mathrm{AC}$ and $\mathrm{PC}$ inoculation enhanced the root length, root volume and root surface area compared to control plants. As the stress increased, only PC inoculation significantly promoted root growth under $0.3 \% \mathrm{NaCl}$. Pan et al. [33] also reported that DSE had positive influences on root morphological characteristics under salt-stress conditions. Moreover, studies have shown that DSE can promote plant root growth under water deficiency and ion stress [50,51]. Extensive and deep roots are conducive to increasing the water and nutrient intake of plants, and eventually influence biomass [31]. 
Thus, the relatively large root length, root volume and root surface area of $A$. ordosica in this study may be beneficial to improve the adaptability of plants to $\mathrm{NaCl}$ stress.

Previous studies have shown that fungal endophytes can produce phytohormones, the process of which is beneficial to host plants to counteract the adverse effects of abiotic stress [52]. IAA, an important plant hormone, plays an important function in plant responses to salt stress [53]. In our study, the content of IAA in PC- and BS-inoculated plant roots was significantly greater than that in the control plants regardless of the $\mathrm{NaCl}$ treatment. The SEM also showed that DSE species was the dominant impact factor and had a significant direct effect on the IAA content in the roots of $A$. ordosica. In previous studies, several DSE species were reported to produce IAA, thereby promoting plant growth and increasing plant stress tolerance $[54,55]$. Qiang et al. [56] reported that inoculation with Alternaria alternata (LQ1230) increased the IAA content, increased the growth of wheat and increased drought tolerance. These results indicated that DSE may also promote the growth of $A$. ordosica by regulating the plant root IAA content, thereby improving the salt stress tolerance of host plants.

An appropriate $\mathrm{Na}^{+}: \mathrm{K}^{+}$ratio in plant tissues represents an important basis for the normal physiological metabolism of plants [8]. Under salt stress, the substitution of $\mathrm{Na}^{+}$for $\mathrm{K}^{+}$in plants often results in an increased $\mathrm{Na}^{+}$content, which in turn damages cells [57]. In the present study, with increasing $\mathrm{NaCl}$ stress, the $\mathrm{Na}^{+}$content and $\mathrm{Na}^{+}: \mathrm{K}^{+}$ratio in the shoots of $A$. ordosica tended to increase. However, no significant changes were observed between inoculated $A$. ordosica (AC, PC) and control plants. These results indicate that DSE did not seem to limit the translocation of $\mathrm{Na}^{+}$to the aboveground plant parts, which agrees with the results of Farias et al. [34]. In addition, the DSE significantly directly affected the $\mathrm{Na}^{+}$ content in the roots of $A$. ordosica. Some DSE promoted the absorption of $\mathrm{K}^{+}$in the roots of $A$. ordosica and decreased the root $\mathrm{Na}^{+}$content regardless of $\mathrm{NaCl}$ stress. The significantly decreased $\mathrm{Na}^{+}$content in the roots inoculated with DSE may be related to DSE promoting the upregulation of RpSOS1 in the roots and increased $\mathrm{Na}^{+}$export to the soil $[57,58]$. Moreover, the SEM analysis showed that root biomass had direct negative effects on the $\mathrm{Na}^{+}$content in the roots. DSE promoted the growth of plant roots and caused a dilution effect, which may be an important reason for the decrease in $\mathrm{Na}^{+}$in the roots [59]. In addition, $\mathrm{G}-$ bacteria had direct negative effects on the $\mathrm{Na}^{+}$content in roots. These findings are consistent with those of Mohamed et al. [33], in which $\mathrm{G}$ - bacteria decreased the $\mathrm{Na}^{+}$in the roots of plants under salt stress. Mendpara et al. [60] also reported that most salt-tolerant bacteria are G bacteria. Studies have shown that bacteria can secrete exopolysaccharides (EPSs) to bind excess $\mathrm{Na}^{+}$ and restrict the influx of $\mathrm{Na}^{+}$into the roots. Moreover, these bacteria can also increase the absorption of $\mathrm{K}^{+}$and the export of $\mathrm{Na}^{+}$by the roots, thus improving plant salt tolerance [61].

Generally, salt stress causes oxidative damage to plant cells, thereby negatively affecting plant growth [62]. In this study, DSE enhanced the antioxidant enzyme activity in the leaves to remove reactive oxygen species (ROS) under salt-stress conditions. SOD, an important antioxidant enzyme, can eliminate ROS generated from oxidative damage [63]. Compared with the control plants, the plants inoculated with AC and BS presented increased SOD activity under $\mathrm{NaCl}$ stress, and the plants inoculated with PC presented 
increased SOD activity only under $0.2 \% \mathrm{NaCl}$. Similar results have been reported by Pan et al. [33], in which DSE increased SOD activity in the leaves of plants under salt stress. As an antioxidant and a ligand peptide, GSH plays an important role in mitigating salt-induced damage [64]. In our study, BS inoculation increased the GSH content of plants under $\mathrm{NaCl}$ stress, and PC inoculation increased the GSH content in plants under $0.1 \% \mathrm{NaCl}$; however, an increase in GSH content in the AC-inoculated plants was detected only under $0.2 \%$ and $0.3 \% \mathrm{NaCl}$. Similar results in which DSE could activate GSH metabolism in Zea mays under heavy metal stress have been reported [65]. These results indicated that, by regulating the accumulation of antioxidants and the $\mathrm{Na}^{+}: \mathrm{K}^{+}$balance, DSE can alleviate the adverse effects of $\mathrm{NaCl}$ stress on plants.

Plant symbiotic fungi normally play important roles in the regulation of the soil microenvironment [12, 66]. Here, DSE had direct negative influences on the soil AP and SOC content but significant direct influences on the soil AN content. BS and PC inoculation reduced the SOC and AP content under $0.1 \%$ and $0.3 \% \mathrm{NaCl}$, and $\mathrm{AC}$ inoculation decreased the SOC content under $0.3 \% \mathrm{NaCl}$. These findings indicated that DSE may regulate the balance of soil nutrients under $\mathrm{NaCl}$ stress. As a bridge between plants and the soil, DSE have been determined to convert soil organic carbon and nitrogen and insoluble phosphorus mineralization into effective forms, which can greatly expand plant organic nutrient pools [67-70]. Moreover, DSE may increase the contact area between plants and the soil through the extension of hyphae, increasing the absorption of nutrients by host plants [22, 23]. In addition, PC inoculation promoted the abundance of $\mathrm{G}$ - bacteria, actinomycetes, fungi and $\mathrm{AM}$ fungi in the soil under $0.2 \%$ and $0.3 \% \mathrm{NaCl}$, and $\mathrm{AC}$ inoculation increased the abundance of soil microbes under $0.3 \% \mathrm{NaCl}$, while BS increased the abundance of soil bacteria and $\mathrm{AM}$ fungi under $0.1 \%$ and $0.2 \% \mathrm{NaCl}$, respectively. These findings indicated that DSE increased the abundance of soil microorganisms under salt stress. Similar results have been reported by He et al. [15], in which DSE increased the abundance of fungi, bacteria and actinomycetes under drought stress. Previous research has also shown that fungi and $\mathrm{G}$ - bacteria have more adaptive capacity to stress environments, and $\mathrm{G}+$ bacteria may positively influence AM fungi propagation $[60,71,72]$. Actinomycetes are also an important part of the rhizosphere microbial community, which can promote plant growth and soil nutrient cycling [73]. In our study, DSE not only significantly directly affected the abundance of fungi and $G+$ bacteria but also indirectly affected the abundance of $\mathrm{G}$ - bacteria and fungi by influencing the SOC content. These results indicated that DSE may release increased amounts of organic nutrients into the soil, thereby promoting the growth of salttolerant microorganisms. In addition, the influence of DSE on the composition and quantity of host plant root exudates may be another factor influencing the microbial community composition. Further studies associated with the effects of DSE on plant growth and soil microflora under salt-stress conditions will help us better understand the ecological functions of DSE in stressful ecosystems.

\section{Conclusion}

In the present study, three DSE isolated from $A$. ordosica could effectively colonize the roots of host plants under different $\mathrm{NaCl}$ concentrations. Although derived from the identical environment, these DSE 
strains exerted considerably different effects on the growth of plants. The effect of DSE inoculation on plant function ranges from beneficial to neutral with increasing $\mathrm{NaCl}$ concentrations and depends on the DSE species and $\mathrm{NaCl}$ treatment. Under $\mathrm{NaCl}$ stress, inoculation with DSE reduced the $\mathrm{Na}^{+}$content and $\mathrm{Na}^{+}: \mathrm{K}^{+}$ratio in plant roots and increased the SOD activity, GSH content and IAA content, as well as the root growth of $A$. ordosica, which alleviated the negative effects of $\mathrm{NaCl}$ stress on the host. DSE also affected the rhizospheric soil microbial community by influencing the status of the SOC, AP and AN contents. Further investigations of the relationship between DSE from different sources and different plants are still needed to confirm the detailed ecological functions under salt-stress conditions.

\section{Methods}

\section{Fungus and plant materials}

Three dark septate endophytes (DSE) isolates were obtained from the roots of Artemisia ordosica grown in the Mu Us Sandy Land of Northwestern China. These DSE were identified as Alternaria chlamydosporigena (AC; MK246176), Paraphoma chrysanthemicola (PC; MF966391), and Bipolaris sorokiniana (BS; MK246180) on the basis of their morphological characteristics and on the basis of sequence analysis of the internal transcribed spacer (ITS) regions of ribosomal DNA (rDNA) [74]. The DSE strains have been deposited in the Plant Ecology Laboratory of Hebei University, China. All DSE strains were maintained in the dark at $27^{\circ} \mathrm{C}$ on potato dextrose agar (PDA) media via subculturing every two weeks.

The seeds of $A$. ordosica were supplied and identified by the Desert Forest Experimental Center, Chinese Academy of Forestry, China. The details of the deposited A. ordosica specimens (PE02235073) were provided by Herbarium, Institute of Botany, Chinese Academy of Sciences. All the seeds were stored at $4{ }^{\circ} \mathrm{C}$ until pregermination.

\section{Plant growth promotion experiment}

A potted plant experiment was conducted in accordance with a randomized complete design $(4 \times 4)$ containing two factors: (1) DSE inoculation treatments, which involved inoculation with $A$. chlamydosporigena (AC), P. chrysanthemicola (PC), and B. sorokiniana (BS) and a noninoculated control (C) treatment; (2) $\mathrm{NaCl}$ stress treatments, which involved concentrations of $0 \%, 0.1 \%, 0.2 \%$ and $0.3 \% \mathrm{NaCl}$ in the soil. Each treatment was replicated five times (three plants/pot/replicate). So there were total 120 experimental pots.

The seeds of $A$. ordosica were sterilized in ethanol (70\%) for $3 \mathrm{~min}$ followed by sodium hypochlorite (2.5\%) for $10 \mathrm{~min}$, after which they were rinsed three times in sterilized water. The sterilized seeds were germinated on $10 \mathrm{~g} / \mathrm{L}$ agar-water media at $27^{\circ} \mathrm{C}$. After that, three seedlings of uniform size were selected and transplanted into a sterile pot $(11.5 \mathrm{~cm}$ height, $13.6 \mathrm{~cm}$ diameter at the top and $9.5 \mathrm{~cm}$ diameter at the base) containing 1,000 g of growth media, which consisted of a 1:1 (v/v) mixture of river sand and soil $(<2 \mathrm{~mm})$. The soil was collected from farmland in North China. The growth media included 
$9.94 \mathrm{mg} / \mathrm{g}$ soil organic carbon (SOC), $16.55 \mathrm{mg} / \mathrm{kg}$ alkaline nitrogen (AN), and $11.26 \mathrm{mg} / \mathrm{kg}$ available phosphorus (AP). With respect to the DSE inoculation treatments, two $9 \mathrm{~mm}$ diameter discs obtained from the margins of an active colony were inoculated within $1 \mathrm{~cm}$ from the roots of $A$. ordosica seedlings. With respect to the noninoculated treatments, two $9 \mathrm{~mm}$ discs were removed from PDA medium without any fungi. All plants were grown in a greenhouse at $27^{\circ} \mathrm{C} / 22^{\circ} \mathrm{C}$ day/night temperature, $14 \mathrm{~h} / 10 \mathrm{~h}$ photoperiod and $60 \%$ mean relative humidity.

Thirty days after inoculation, $\mathrm{NaCl}$ stress treatments were imposed by the application of $\mathrm{NaCl}$ solutions to the pots. To avoid osmotic shock, $\mathrm{NaCl}$ stress was applied in increments [75]; that is, each pot received $50 \mathrm{~mL}$ of $0.34 \mathrm{~mol} / \mathrm{L} \mathrm{NaCl}$ solutions at 5-day intervals successively. Fifty milliliters of sterile water without $\mathrm{NaCl}$ was applied to the controls each time to ensure consistent soil moisture. $\mathrm{NaCl}$ solutions were applied such that the desired $0 \%, 0.1 \%, 0.2 \%$ and $0.3 \% \mathrm{NaCl}$ levels were reached [76]. Finally, the plants were harvested after 60 days of treatment.

\section{Plant biomass and morphological parameters}

Prior to harvest, the plant height and shoot branching in each replicate (three plants/pot) were recorded. The roots and shoots in each replicate were subsequently harvested separately. The cleaned roots were scanned utilizing an Epson Perfection V800 Photo scanner. The root morphological parameters, including root length, root volume, root diameter and root surface area, were measured using the WinRHIZO image analysis system (Regent Instruments, Quebec, Canada). Fresh roots were subsequently collected and used to estimate the DSE colonization levels and indoleacetic acid (IAA) concentration. Similarly, fresh leaves were used to measure physiological parameters, such as superoxide dismutase (SOD) activity and glutathione (GSH) content. The remaining fresh shoots and roots were dried to a constant weight at $70{ }^{\circ} \mathrm{C}$ to confirm the plant biomass and the $\mathrm{Na}^{+}$and $\mathrm{K}^{+}$contents. Soil samples from each pot were sieved (2$\mathrm{mm}$ mesh size) and then split into two parts: one subsample was stored at $-80{ }^{\circ} \mathrm{C}$ to analyze the community composition of soil microbes, and the other subsample was dried at room temperature to analyze soil physicochemical properties.

\section{DSE colonization analyses}

Fresh roots of $A$. ordosica were randomly sampled from each pot to determine DSE colonization levels. First, the roots were cleaned under running water. The root segments $(0.5 \mathrm{~cm})$ were clarified in potassium hydroxide $(10 \%, \mathrm{w} / \mathrm{v})$ and then dyed in acid fuchsin $(0.5 \%, \mathrm{w} / \mathrm{v})$ according to the methods of Phillips and Hayman [77]. Thirty root segments of each sample were randomly selected and pressed onto slides, and then observed under a light microscope (Olympus BX51) with $20 \times$ and $40 \times$ eyepieces [78]. The DSE total colonization rate (\%) was expressed as the percentage of infected root segments per root sample.

\section{Determination of SOD activity and GSH content}

The activity of SOD in the leaves was assayed by measuring the diminution in the opticaldelnsity of the nitro blue tetrazolium (NBT) complex according to Elavarthi and Martin [79]. Briefly, fresh leaves $(0.2 \mathrm{~g})$ were ground together with phosphate buffered saline (PBS) $(50 \mathrm{mM}, 5 \mathrm{~mL} ; \mathrm{pH} 7.8)$ in an ice bath and 
then centrifuged for $10 \mathrm{~min}$ at $4,000 \times \mathrm{g}$ and $4{ }^{\circ} \mathrm{C}$. The reaction solution $(5 \mathrm{~mL})$ contained $0.1 \mathrm{~mL}$ of enzyme extract, $30 \mathrm{~mL}$ of riboflavin $(33 \mu \mathrm{mol} / \mathrm{L}), 0.3 \mathrm{~mL}$ of NBT $(1.25 \mathrm{mmol} / \mathrm{L}), 0.3 \mathrm{~mL}$ of methionine $(220 \mathrm{mmol} / \mathrm{L})$ and $4 \mathrm{~mL}$ of PBS (50 mM). One unit of SOD was defined as enzyme activity that inhibited NBT reduction by $50 \%$ at $560 \mathrm{~nm}$.

The GSH content was assayed according to the dithiobis-nitrobenzoic acid (DTNB) method described by Anderson [80]. Briefly, fresh leaves $(0.2 \mathrm{~g})$ were ground together with sulfosalicylic acid $(5 \%, \mathrm{w} / \mathrm{v})$ at $4{ }^{\circ} \mathrm{C}$. The crude extract was subsequently centrifuged for $10 \mathrm{~min}$ at $10,000 \times \mathrm{g}$. The reaction mixture consisted of PBS $(100 \mathrm{mM}, 0.6 \mathrm{~mL})$, DTNB $(40 \mu \mathrm{L})$ and the supernatant $(0.5 \mathrm{~mL})$. The opticaldelnsity of the supernatant was determined at $421 \mathrm{~nm}$. The standard curve was used to calculate GSH content.

\section{Measurement of IAA content}

The IAA content in the roots was estimated by an IAA ELISA Kit (MLBIO, Shanghai, China). Fresh roots (approximately $100 \mathrm{mg}$ ) were homogenized in a precooled mortar $\left(4^{\circ} \mathrm{C}\right)$ containing PBS $(0.01 \mathrm{~mol} / \mathrm{L}$, $1 \mathrm{~mL} ; \mathrm{pH}=7.2-7.4)$. The supernatant was collected after centrifugation at $3,000 \times \mathrm{g}$ for $20 \mathrm{~min}$ at $4{ }^{\circ} \mathrm{C}$. The supernatant $(10 \mu \mathrm{L})$ was used to determine the IAA content, and the procedures were conducted in accordance with the instructions provided by the manufacturer. The absorbance was detected by the use of an Epoch 2 microplate reader (BioTek, Winooski, USA) at a wavelength of $450 \mathrm{~nm}$. The IAA content in the roots was determined in terms of the standard curve.

\section{Determination of $\mathrm{Na}^{+}$and $\mathrm{K}^{+}$contents}

Dried powdery samples (approximately $0.1 \mathrm{~g}$ ) of shoots and roots were immersed in $\mathrm{HNO}_{3}: \mathrm{HClO}_{4}(3: 1$, $\mathrm{v} / \mathrm{v}$ ) solution and heated to $220^{\circ} \mathrm{C}$ for digestion to extract $\mathrm{Na}^{+}$and $\mathrm{K}^{+}$ions. The cooled digestion solution was diluted to $25 \mathrm{~mL}$ by adding deionized water, after which the $\mathrm{Na}^{+}$and $\mathrm{K}^{+}$contents were measured by inductively coupled plasma optical emission spectrometry (ICP-OES; Beijing Ruiguang Technology Co., Ltd., China).

\section{Soil physicochemical properties analysis}

The SOC content was measured according to the potassium dichromate oxidation method [81, 82]. A dried soil sample (approximately $1 \mathrm{~g}$ ) was placed in a tube containing $\mathrm{K}_{2} \mathrm{Cr}_{2} \mathrm{O}_{7}$ solution $(0.8 \mathrm{~mol} / \mathrm{L}, 5 \mathrm{~mL})$ and $\mathrm{H}_{2} \mathrm{SO}_{4}(98 \%, 5 \mathrm{~mL})$ and then boiled for $5 \mathrm{~min}$ at $170{ }^{\circ} \mathrm{C}$. After cooling, the solution was transferred to a conical flask, and the volume was adjusted to $60 \mathrm{~mL}$ by dilution with distilled deionized water. The excess dichromate was subsequently titrated with $0.2 \mathrm{~mol} / \mathrm{L} \mathrm{FeSO}_{4}$ with diphenylamine used as an indicator.

The AN content was determined according to the alkaline hydrolysis diffusion method [82]. Approximately $2 \mathrm{~g}$ of dried soil sample was uniformly distributed in the outer chamber of the diffusion dish. $\mathrm{H}_{3} \mathrm{BO}_{3}$ indicator $(20 \mathrm{~g} / \mathrm{L}, 2 \mathrm{~mL})$ and $\mathrm{NaOH}(1 \mathrm{~mol} / \mathrm{L}, 10 \mathrm{~mL})$ were added to the inner and outer chambers, respectively. The diffusion dishes were then sealed and incubated at $40{ }^{\circ} \mathrm{C}$ for $24 \mathrm{~h}$. Titration was subsequently performed via $0.01 \mathrm{~mol} / \mathrm{L} \mathrm{H}_{2} \mathrm{SO}_{4}$ solution. 
The AP content was measured by the chlorostannus-reduced molybdophosphoric blue color method according to the methods of Olsen et al. [83]. Briefly, dried soil samples (approximately $1 \mathrm{~g}$ ) were placed into a conical flask containing both $\mathrm{NaHCO}_{3}$ solution $(0.5 \mathrm{~mol} / \mathrm{L}, 50 \mathrm{~mL})$ and phosphor-free activated carbon $(0.1 \mathrm{~g})$. After the mixture had been shaken for $30 \mathrm{~min}$, the filtrate $(10 \mathrm{~mL})$ and molybdenum vanadate solution $(5 \mathrm{~mL})$ were mixed together, followed by dilution with distilled deionized water to $50 \mathrm{~mL}$. The mixed reagent was then nurtured for $30 \mathrm{~min}$, after which the absorption values were determined at $700 \mathrm{~nm}$ via a spectrometer.

\section{Soil microbial community composition}

The composition of the rhizosphere soil microbial community was determined by phospholipid fatty acid (PLFA) analysis. Briefly, soil subsamples (approximately $8.0 \mathrm{~g}$, frozen) were placed into a tube containing $23 \mathrm{~mL}$ of chloroform:methanol:phosphate buffer (1:2:0.8 v/v/v) solution to extract lipids according to the method of Bossio and Scow [84]. The extracts were separated by continuous elution with chloroform $(5 \mathrm{~mL})$, acetone $(20 \mathrm{~mL})$ and methanol $(5 \mathrm{~mL})$ on silica acid columns. Then, the organic solvent was evaporated using a nitrogen blowing instrument. The phospholipids were sequentially saponified and methylated to form fatty acid methyl esters (FAMEs).

The gas chromatograph (Agilent $6890 \mathrm{~N}$ ) in conjunction with MIDI software (MIDI, Newark, Delaware, USA) was used to identify and quantify the extracted FAMEs. The software package Sherlock MIS 4.5 could automatically control the gas chromatograph for calibration, subsequent sample sequencing, naming, and peak integration operations. The mixture of straight-chain saturated and hydroxy FAMEs with a length of 10-20 carbons (MIDI Part No. 1208) was used as the calibration standard. Gram-positive $(\mathrm{G}+)$ bacterial biomarkers included $14: 1$ iso $\mathrm{w} 7 \mathrm{c}, 14: 0$ iso, $14: 0$ anteiso, $15: 1$ iso $w 9 \mathrm{c}$, and 15:1 iso w6c, and gram-negative $\left(\mathrm{G}^{-}\right)$bacterial biomarkers included 14:1 w9c, 14:1 w8c, 14:1 w7c, 14:1 w5c, 15:1 w9c, 15:1 w8c, 15:1 w 7c, and 15:1 w6c. The actinomycete biomarkers included 16:0 10-methyl, 17:1 w7c 10methyl, 17:0 10-methyl, and 18:1 w7c 10-methyl. The fungal biomarkers contained 18:2 w6c and 18:1 w9c. The fatty acid 16:1 w5c was determined to be a biomarker for AM fungi [15].

\section{Statistical analysis}

Two-way ANOVA was applied to evaluate the influences of DSE inoculation, $\mathrm{NaCl}$ stress and interactions on biomass production, morphological characteristics, physiological parameters, soil factors and microbial community composition in the rhizospheric soil of each plant. The partial eta squared $\left(\mathrm{n}_{\mathrm{p}}{ }^{2}\right)$ value represented the effect size of the different factors. Duncan's multivariate range test was used to assess the differences in means between the different treatments $(P<0.05)$. Structural equation modeling (SEM) was constructed to determine the causal relationships between DSE species, $\mathrm{NaCl}$ stress and soil factors on the growth of $A$. ordosica and the rhizospheric microbial composition via AMOS 21.0. Before constructing the SEM, Spearman's correlation analysis was conducted to evaluate the interrelationships among the variables via SPSS 19.0 software. The statistical analysis and figure plotting were performed by SPSS 19.0, AMOS 21.0 and Kaleida Graph 4.5. 


\section{Abbreviations}

DSE: Dark septate endophytes; PC: Paraphoma chrysanthemicola; BS: Bipolaris sorokiniana; AC: Alternaria chlamydosporigena; C: Noninoculated control; SOD: Superoxide dismutase; GSH: Glutathione; IAA: Indoleacetic acid; SOC: Soil organic carbon; AP: Available phosphorus; AN: Alkaline nitrogen; AM: Arbuscular mycorrhizal; $\mathrm{G}$ - bacteria: Gram-negative bacteria; $\mathrm{G}+$ bacteria: Gram- positive bacteria; SEM: Structural equation modeling; TLI: Tacker-Lewis index; CFI: Comparative fit index; RMSEA: Root mean square error of approximation; EPSs: Exopolysaccharides; ROS: Reactive oxygen species; ITS: Internal transcribed spacer; PDA: Potato dextrose agar; NBT: Nitro blue tetrazolium; PBS: Phosphate buffered saline; DTNB: Dithiobis-nitrobenzoic acid; PLFA: Phospholipid fatty acid; NLFAs: Neutral lipid fatty acids; FAMEs: Fatty acid methyl esters; $\eta_{\mathrm{p}}{ }^{2}$ : The partial eta squared

\section{Declarations}

\section{Acknowledgments}

The authors are grateful to the students Xue Zhang, Yedong Li, Wanyun Li, Jingya Yang, Xin Zhao and Jingxin Shi of Hebei University for laboratory work. Additionally, we also thank American Journal Experts for providing language editing services.

\section{Authors' Contributions}

$\mathrm{LH}, \mathrm{LZ}$ and $\mathrm{XH}$ conceived and designed the experiments. $\mathrm{LH}, \mathrm{YZ}$ and $\mathrm{DZ}$ performed the experiments. $\mathrm{LH}$ analyzed the data. $\mathrm{XH}, \mathrm{XL}$ and $\mathrm{LH}$ wrote the manuscript. $\mathrm{LH}$ and $\mathrm{XL}$ contributed equally to this manuscript. All authors read and approved the final manuscript.

\section{Funding}

This study was financially supported by the National Natural Science Foundation of China (No. 31770561, 31800345).

\section{Availability of data and materials}

The datasets used and/or analyzed during the current study are available from the corresponding author on reasonable request.

\section{Ethics approval and consent to participate}


A potted plant experiment in this study was performed in a greenhouse. We confirmed that the sampling did not involve any endangered or protected plant species and declare that the work reported here complies with the current laws of China and the IUCN Policy Statement on Research Involving Species at Risk of Extinction.

\section{Consent to publish}

Not applicable.

\section{Competing interests}

The authors declare that they have no competing interests.

\section{Authors' information}

${ }^{1}$ College of Life Sciences, Hebei University, No. 180, Wusidong Rd., Baoding 071002, China. ${ }^{2}$ Institute of Life Sciences and Green Development, Hebei University, No. 180, Wusidong Rd., Baoding 071002, China

\section{References}

1. Yuan BC, Li ZZ, Liu H, Gao M, Zhang YY. Microbial biomass and activity in salt affected soils under arid conditions. Appl Soil Ecol. 2007; 35: 319-328.

2. Pannell DJ, Ewing MA. Managing secondary dryland salinity: options and challenges. Agr Water Manag. 2006; 80: 41-56.

3. Li JG, Pu LJ, Han MF, Zhu M, Zhang RS, Xiang YZ, et al. Soil salinization research in China: advances and prospects. J Geogr Sci. 2014; 24: 943-960.

4. Sheng $M$, Tang $M$, Zhang FF, Huang $\mathrm{YH}$. Influence of arbuscular mycorrhiza on organic solutes in maize leaves under salt stress. Mycorrhiza. 2011; 21: 423-430.

5. Porcel R, Aroca R, Ruiz-Lozano JM. Salinity stress alleviation using arbuscular mycorrhizal fungi. a review. Agron Sustain Dev. 2012; 32: 181-200.

6. Wu N, Li Z, Wu F, Tang M. Comparative photochemistry activity and antioxidant responses in male and female Populus cathayana cuttings inoculated with arbuscular mycorrhizal fungi under salt stress. Sci Rep. 2016; 6: 37663.

7. Becker D, Hoth S, Ache P, Wenkel S, Roelfsema MRG, Meyerhoff $O$, et al. Regulation of the ABAsensitive Arabidopsis potassium channel gene GORK in response to water stress. FEBS Lett. 2003; 554: 119-126.

8. Munns R, Tester M. Mechanisms of salinity tolerance. Annu Rev Plant Biol. 2008; 59: 651-681. 
9. Gill SS, Tuteja N. Reactive oxygen species and antioxidant machinery in abiotic stress tolerance in crop plants. Plant Physiol Bioch. 2010; 48: 909-930.

10. Pollastri S, Savvides A, Pesando M, Lumini E, Volpe MG, Ozudogru EA, et al. Impact of two arbuscular mycorrhizal fungi on Arundo donax response to salt stress. Planta. 2017; 247: 573-585.

11. Santos-Medellín C, Edwards J, Liechty Z, Nguyen B, Sundaresan V. Drought stress results in a compartment-specific restructuring of the rice root-associated microbiomes. mBio. 2017; 8: e00764-

12. Zhang S, Gan Y, Xu B. Application of plant-growth-promoting fungi Trichoderma longibrachiatum T6 enhances tolerance of wheat to salt stress through improvement of antioxidative defense system and gene expression. Front in Plant Sci. 2016; 07: 1405.

13. Hol WHG, de Boer W, Termorshuizen AJ, Meyer KM, Schneider JHM, van Dam NM, et al. Reduction of rare soil microbes modifies plant-herbivore interactions. Ecol Lett. 2010; 13: 292-

14. Lau JA, Lennon JT. Evolutionary ecology of plant-microbe interactions: soil microbial structure alters selection on plant traits. New Phytol. 2011; 192: 215-

15. He C, Wang WQ, Hou JL. Plant growth and soil microbial impacts of enhancing licorice with inoculating dark septate endophytes under drought stress. Front Microbiol. 2019a; 10: 2277.

16. Yang YR, Cao YP, Li ZX, Songtao AZ, Wang JL, Tang ZH, et al. Interactive effects of exogenous melatonin and Rhizophagus intraradices on saline-alkaline stress tolerance in Leymus chinensis. Mycorrhiza. 2020; 30: 357-371.

17. Deja-Sikora E, Kowalczyk A, Trejgell A, Szmidt-Jaworska A, Baum C, Mercy L, et al. Arbuscular mycorrhiza changes the impact of potato virus $Y$ on growth and stress tolerance of Solanum tuberosum in vitro. Front Microbiol. 2019; 10: 2971.

18. Lin HF, Xiong J, Zhou HM, Chen CM, Lin MZ, Xu XM, et al. Growth promotion and disease resistance induced in Anthurium colonized by the beneficial root endophyte Piriformospora indica. BMC Plant Biol. 2019; 19: 40.

19. Jumpponen A, Trappe JM. Dark septate endophytes: a review of facultative biotrophic rootcolonizing fungi. New Phytol. 1998; 140: 295-310.

20. Andrade-Linares DR, Grosch R, Franken P, Karl-Heinz-Rexer, Kost G, Restrepo S, et al. Colonization of roots of cultivated Solanum lycopersicum by dark septate and other ascomycetous endophytes. Mycologia. 2011; 103: 710-721.

21. Sieber TN, Grünig CR. Fungal root endophytes. In: Eshel A, Beeckman T, editors. Plant roots-the hidden half, fourth ed. Florida: CRC Press; 2013. p. 38-49.

22. Mandyam K, Jumpponen A. Seeking the elusive function of the root-colonising dark septate endophytic fungi. Stud Mycol. 2005; 53: 173-189.

23. Addy HD, Piercey MM, Currah RS. Microfungal endophytes in roots. Can J Bot. 2005; 83: 1-13.

24. Sonjak S, Udovič M, Wraber T, Likar M, Regvar M. Diversity of halophytes and identification of arbuscular mycorrhizal fungi colonising their roots in an abandoned and sustained part of Sečovlje salterns. Soil Biol Biochem. 2009; 41: 1847-1856. 
25. Lugo MA., Reinhart KO., Menoyo E, Crespo EM, Urcelay C. Plant functional traits and phylogenetic relatedness explain variation in associations with root fungal endophytes in an extreme arid environment. Mycorrhiza. 2015; 25: 85-95.

26. Vohník M, Borovec $\mathrm{O}$, Kolařík M. Communities of cultivable root mycobionts of the seagrass Posidonia oceanica in the northwest mediterranean sea are dominated by a hitherto undescribed Pleosporalean dark septate endophyte. Microb Ecol. 2016; 71: 442-451.

27. Berthelot C, Leyval C, Foulon J, Chalot M, Blaudez D. Plant growth promotion, metabolite production and metal tolerance of dark septate endophytes isolated from metal-polluted poplar phytomanagement sites. FEMS Microbiol Ecol. 2016; 92: fiw144.

28. Xie LL, He XL, Wang K, Hou LF, Sun Q. Spatial dynamics of dark septate endophytes in the roots and rhizospheres of Hedysarum scoparium in northwest China and the influence of edaphic variables. Fungal Ecol. 2017; 26: 135-143.

29. Newsham KK. A meta-analysis of plant responses to dark septate root endophytes. New Phytol. 2011; 190: 783-793.

30. Barrow JR. Atypical morphology of dark septate fungal root endophytes of Bouteloua in arid southwestern USA rangelands. Mycorrhiza. 2003; 13: 239-247.

31. Li X, He XL, Hou LF, Ren Y, Wang SJ, Su F. Dark septate endophytes isolated from a xerophyte plant promote the growth of Ammopiptanthus mongolicus under drought condition. Sci Rep. 2018; 8:7896.

32. He C, Wang WQ, Hou JL. Characterization of dark septate endophytic fungi and improve the performance of licorice under organic residue treatment. Front Microbiol. 2019b; 10:1364.

33. Pan XY, Qin Y, Yuan ZL. Potential of a halophyte-associated endophytic fungus for sustaining Chinese white poplar growth under salinity. Symbiosis. 2018; 76: 109-116.

34. Farias GC, Nunes KG, Soares MA, de Siqueira KA, Lima W C, Neves ALR, et al. Dark septate endophytic fungi mitigate the effects of salt stress on cowpea plants. Braz J Microbiol. 2019; 51: 243-253.

35. Mateu MG, Baldwin AH, Maul JE, Yarwood SA. Dark septate endophyte improves salt tolerance of native and invasive lineages of Phragmites australis. ISME J. 2020; 14: 1943-1954.

36. Yuan ZL, Druzhinina IS, Labbé J, Redman R, Qin Y, Rodriguez R, et al. Specialized microbiome of a halophyte and its role in helping non-host plants to withstand salinity. Sci Rep. 2016; 6: 32467.

37. Bai Y, Muller DB, Srinivas G, Garrido-Oter R, Potthoff E, Rott M, et al. Functional overlap of the arabidopsis leaf and root microbiota. Nature. 2015; 528: 364-369.

38. Hashem A, Abd_Allah EF, Alqarawi AA, Al-Huqail AA, Wirth S, Egamberdieva D. The interaction between arbuscular mycorrhizal fungi and endophytic bacteria enhances plant growth of Acacia gerrardii under salt stress. Front microbiol. 2016; 7: 1089.

39. Yang HX, Zhang JT, Li ZD, Wu B, Zhang ZS, Wang Y. Comparative study on spatial patterns of the Artemisia ordosica population in the Mu Us sandy land. Acta Ecol Sin. 2008; 28: 1901-1910 
40. Xiang YL, Xiang YX, Wang LP. Electron beam irradiation to enhance enzymatic saccharification of alkali soaked Artemisia ordosica used for production of biofuels. J Environ Chem Eng. 2017; 5: 40934100.

41. Zhang T, Hu YJ, Zhang K, Tian CY, Guo JX. Arbuscular mycorrhizal fungi improve plant growth of Ricinus communis by altering photosynthetic properties and increasing pigments under drought and salt stress. Ind Crop Prod. 2018; 117: 13-19.

42. Peterson RL, Wagg C, Pautler M. Associations between microfungal endophytes and roots: do structural features indicate function?. Botany. 2008; 86: 445-456.

43. Orlowska E, Przybylowicz W, Orlowski D, Turnau K, Mesjasz-Przybylowicz J. The effect of mycorrhiza on the growth and elemental composition of Ni-hyperaccumulating plant Berkheya coddii Environ Pollut. 2011; 159: 3730-3738.

44. Xu RB, Li T, Cui HL, Wang JL, Yu X, Ding YH, et al. Diversity and characterization of Cd-tolerant dark septate endophytes (DSEs) associated with the roots of Nepal alder (Alnus nepalensis) in a metal mine tailing of southwest China. Appl Soil Ecol. 2015; 93: 11-18.

45. Ban YH, Xu ZY, Yang YR, Zhang HH, Chen $\mathrm{H}$, Tang M. Effect of Dark septate endophytic fungus gaeumannomyces cylindrosporus on plant growth, photosynthesis and $\mathrm{Pb}$ tolerance of maize (Zea mays). Pedosphere. 2017; 27: 283-292.

46. Li X, He C, He XL, Su F, Hou LF, Ren Y, et al. Dark septate endophytes improve the growth of host and non-host plants under drought stress through altered root development. Plant Soil. 2019; 439: 259272.

47. Ban YH, Tang M, Chen H, Xu ZY. The response of dark septate endophytes (DSE) to heavy metals in pure culture. Plos One. 2012; 7: e47968.

48. Rahnama A, Munns R, Poustini K, Watt M. A screening method to identify genetic variation in root growth response to a salinity gradient. J Exp Bot. 2011; 62: 69-77.

49. Shelden MC, Dias DA, Jayasinghe NS, Bacic A, Roessner U. Root spatial metabolite profiling of two genotypes of barley (Hordeum vulgare) reveals differences in response to short-term salt stress. J Exp Bot. 2016; 67: 3731-3745.

50. Liu Y, Wei XL. Dark septate endophyte improves drought tolerance of Ormosia hosiei Hemsley \& E. H. Wilson by modulating root morphology, ultrastructure, and the ratio of root hormones. Forests. 2019; 10: 830.

51. Hou LF, Yu J, Zhao LL, He XL. Dark septate endophytes improve the growth and the tolerance of Medicago sativa and Ammopiptanthus mongolicus under cadmium stress. Front. Microbiol. 2020; 10:3061.

52. Khan AL, Hamayun M, Kang SM, Kim YH, Jung HY, Lee JH, et al. Endophytic fungal association via gibberellins and indole acetic acid can improve plant growth under abiotic stress: an example of Paecilomyces formosus BMC Microbiol. 2012; 12: 3.

53. Sarwat M, Hashem A, Ahanger MA, Abd_Allah EF, Alqarawi AA, Alyemeni MN, et al. Mitigation of $\mathrm{NaCl}$ stress by arbuscular mycorrhizal fungi through the modulation of osmolytes, antioxidants and 
secondary metabolites in mustard (Brassica juncea) plants. Front Plant Sci. 2016; 7: 869.

54. Waqas M, Khan AL, Kamran M, Hamayun M, Kang SM, Kim YH, et al. Endophytic fungi produce gibberellins and indoleacetic acid and promotes host-plant growth during stress. Molecules. 2012; 17: $10754-10773$.

55. Priyadharsini P, Muthukumar T. The root endophytic fungus Curvularia geniculata from Parthenium hysterophorus roots improves plant growth through phosphate solubilization and phytohormone production. Fungal Ecol. 2017; 27: 69-77.

56. Qiang XJ, Ding JJ, Lin W, Li QZ, Xu CY, Zheng Q, et al. Alleviation of the detrimental effect of water deficit on wheat (Triticum aestivum) growth by an indole acetic acid-producing endophytic fungus. Plant Soil. 2019; 439: 373-391.

57. Abdelaziz ME, Kim D, Ali S, Fedoroff NV, Ai-Babili S. The endophytic fungus Piriformospora indica enhances Arabidopsis thaliana growth and modulates $\mathrm{Na}^{+} / \mathrm{K}^{+}$homeostasis under salt stress conditions. Plant Sci. 2017; 263: 107-115.

58. Shi H, Quintero FJ, Pardo JM, Zhu JK. The putative plasma membrane $\mathrm{Na}^{+} / \mathrm{H}^{+}$antiporter SOS1 controls long-distance $\mathrm{Na}^{+}$transport in plants. Plant Cell. 2002; 14: 465-477.

59. Amanifar S, Khodabandeloo M, Fard EM, Askari MS, Ashrafi M. Alleviation of salt stress and changes in glycyrrhizin accumulation by arbuscular mycorrhiza in liquorice (Glycyrrhiza glabra) grown under salinity stress. Environ Exp Bot. 2019; 160: 25-34.

60. Mendpara J, Parekh V, Vaghela S, Makasana A, Kunjadia PD, Sanghvi G, et al. Isolation and characterization of high salt tolerant bacteria from agricultural soil. Eur J Exp Biol. 2013; 3: 351-358.

61. Qin Y, Druzhinina IS, Pan XY, Yuan ZL. Microbially mediated plant salt tolerance and microbiomebased solutions for saline agriculture. Biotechnol Adv. 2016; 34: 1245-1259.

62. Deinlein U, Stephan AB, Horie T, Luo W, Xu GH, Schroeder J. Plant salt-tolerance mechanisms. Trends in Plant Sci. 2014; 19: 371-379.

63. Collin-Hansen C, Andersen RA, Steinnes E. Molecular defense systems are expressed in the king bolete (Boletus edulis) growing near metal smelters. Mycologia. 2005; 97: 973-983.

64. Chen JH, Jiang HW, Hsieh EJ, Chen HY, Chien CT, Hsieh HL, et al. Drought and salt stress tolerance of an Arabidopsis glutathione S-transferase U17 knockout mutant are attributed to the combined effect of glutathione and abscisic acid. Plant Physiol. 2012; 158: 340-351.

65. Zhan FD, Li B, Jiang M, Qin L, Wang JX, He YM, et al. Effects of a root-colonized dark septate endophyte on the glutathione metabolism in maize plants under cadmium stress. J Plant Interact. 2017; 12: 421-428.

66. Xu J, Liu SJ, Song SR, Guo HL, Tang JJ, Yong JWH, et al. Arbuscular mycorrhizal fungi influence decomposition and the associated soil microbial community under different soil phosphorus availability. Soil Biol Biochem. 2018; 120: 181-190.

67. Knapp DG, Kovács GM. Interspecific metabolic diversity of root colonizing endophytic fungi revealed by enzyme activity tests. FEMS Microbiol Ecol. 2016; 92: fiw190. 
68. Surono, Kazuhiko N. The dark septate endophytic fungus Phialocephala fortinii is a potential decomposer of soil organic compounds and a promoter of Asparagus officinalis Fungal Ecol. 2017; 28: 1-10.

69. Spagnoletti FN, Tobar NE, Pardo AFD, Chiocchio VM, Lavado RS. Dark septate endophytes present different potential to solubilize calcium, iron and aluminum phosphates. Appl Soil Ecol. 2017; 111: 25-32.

70. Vergara C, Araujo KEC, Urquiaga S, Schultz N, Balieiro FdC, Medeiros PS, et al. Dark septate endophytic fungi help tomato to acquire nutrients from ground plant material. Front Microbiol. 2017; 8: 2437.

71. Artursson, V, Finlay, RD., Jansson, JK. Interactions between arbuscular mycorrhizal fungi and bacteria and their potential for stimulating plant growth. Environ. Microbiol. 2006; 8: 1-10.

72. Carrasco L, Gattinger A, Fliessbach A, Roldan A, Schloter M, Caravaca F. Estimation by PLFA of microbial community structure associated with the rhizosphere of Lygeum spartum and Piptatherum miliaceum growing in semiarid mine tailings. Microb Ecol. 2010; 60: 265-271.

73. Sreevidya M, Gopalakrishnan S, Kudapa H, Varshney RH. Exploring plant growth-promotion actinomycetes from vermicompost and rhizosphere soil for yield enhancement in chickpea. Braz J Microbiol. 2016; 47: 85-95.

74. Hou LF, He XL, Li X, Wang SJ, Zhao LL. Species composition and colonization of dark septate endophytes are affected by host plant species and soil depth in the Mu Us sandland, northwest China. Fungal Ecol. 2019; 39: 276-284.

75. Estrada B, Aroca R, Barea JM, Ruiz-Lozano JM. Native arbuscular mycorrhizal fungi isolated from a saline habitat improved maize antioxidant systems and plant tolerance to salinity. Plant Sci. 2013; 201-202: 42-51.

76. Yang XJ, Dong M, Huang ZY. Role of mucilage in the germination of Artemisia sphaerocephala (Asteraceae) achenes exposed to osmotic stress and salinity. Plant Physiol Bioch. 2010; 48: 131-135.

77. Phillips JM, Hayman DS. Improved procedures for clearing roots and staining parasitic and vesiculararbuscular mycorrhizal fungi for rapid assessment of infection. Trans Br Mycol Soc. 1970; 55: 158163.

78. Biermann B, Linderman RG. Quantifying vesicular-arbuscular mycorrhizae: a proposed method towards standardization. New Phytol. 1981; 87: 63-67.

79. Elavarthi S, Martin B. Spectrophotometric assays for antioxidant enzymes in plants. New York: Humana Press; 2010. 639: 273-280.

80. Anderson ME. Determination of glutathione and glutathione disulfide in biological samples. Method Enzymol. 1985; 113:548-555.

81. Rowell DL. Soil Science: Methods and Applications. London: Longman Group, UK Ltd; 1994.

82. Bao SD. Agrochemical analysis of soil. Beijing: Chinese Agricultural Press; 2000. p. 44-49. 
83. Olsen SR, Cole CV, Watanabe FS, Dean LA. Estimation of available phosphorus in soils by extraction with sodium bicarbonate. USDA circular 939. Washington DC: US Department of Agriculture; 1954. p. 1-18.

84. Bossio DA, Scow KM. Impacts of carbon and flooding on soil microbial communities, phospholipid fatty acid profiles and substrate utilization patterns. Microbial Ecol. 1998; 35: 265-278.

\section{Figures}

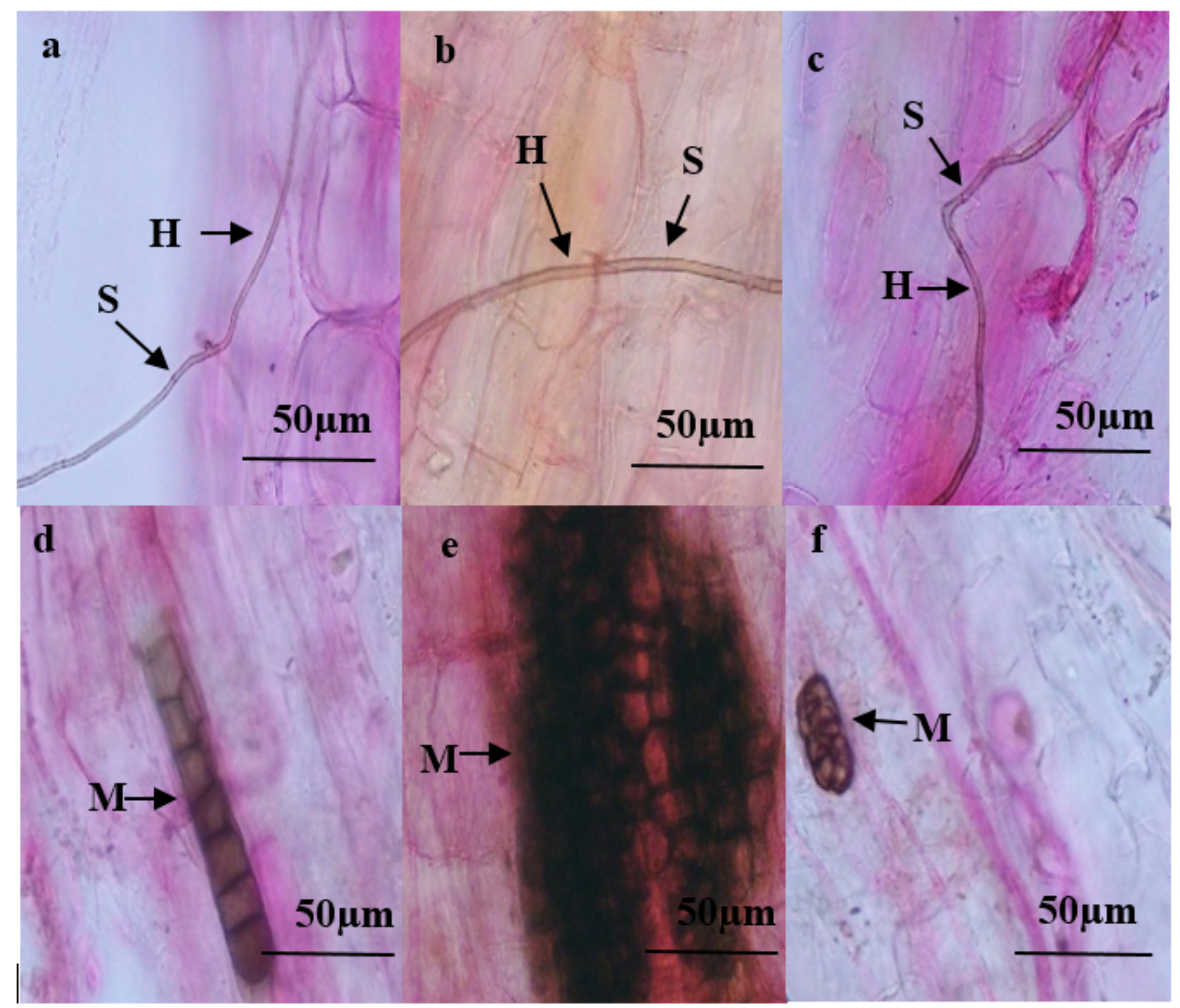

\section{Figure 1}

Colonization of dark septate endophytes (DSE) in the roots of inoculated Artemisia ordosica. Arrows indicate: $\mathrm{H}=\mathrm{DSE}$ hyphae, M=DSE microsclerotia, S= DSE hyphal septa. Scale bars=50 $\mu \mathrm{m}$. 


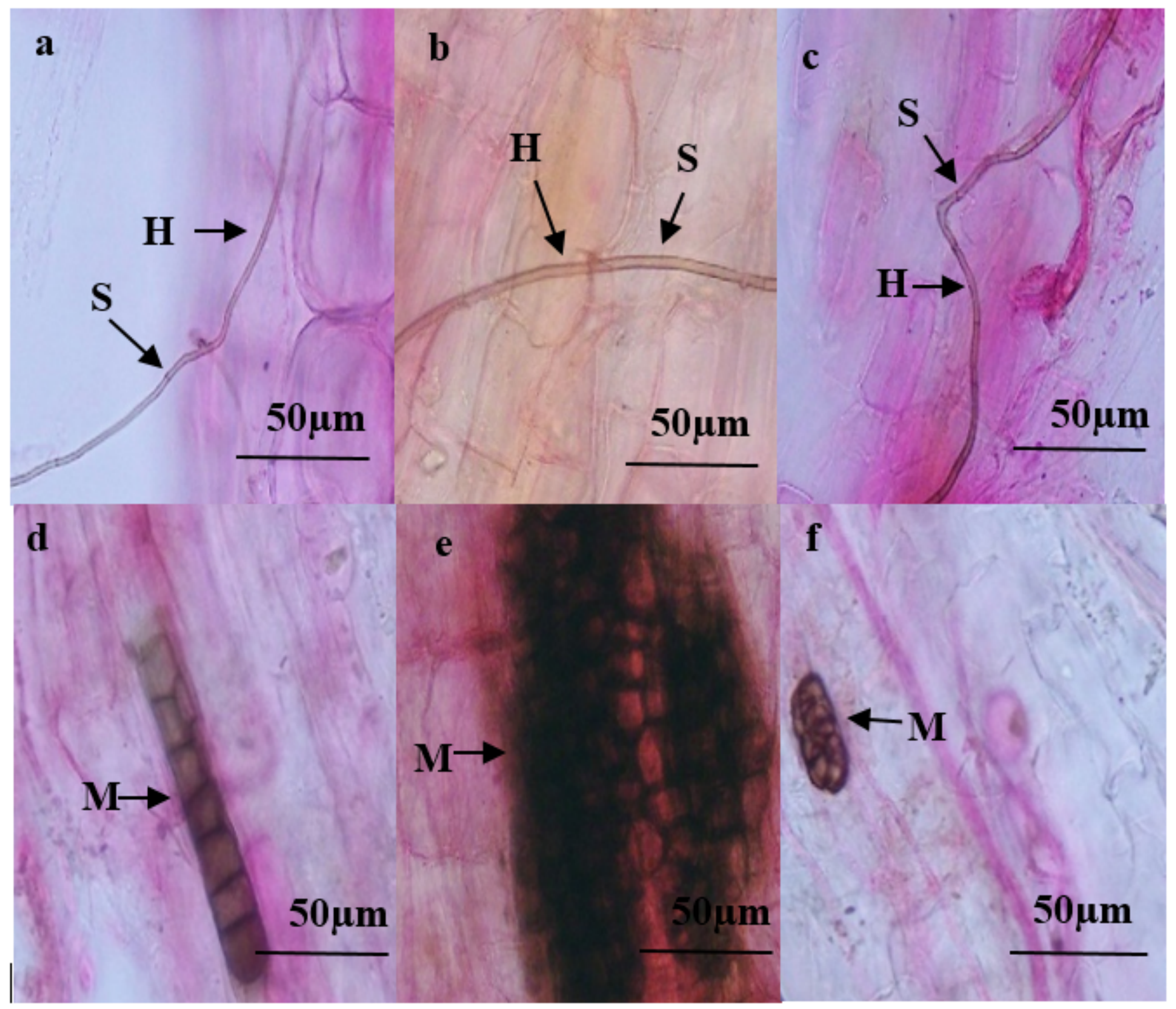

Figure 1

Colonization of dark septate endophytes (DSE) in the roots of inoculated Artemisia ordosica. Arrows indicate: H=DSE hyphae, M=DSE microsclerotia, S= DSE hyphal septa. Scale bars=50 $\mu \mathrm{m}$. 


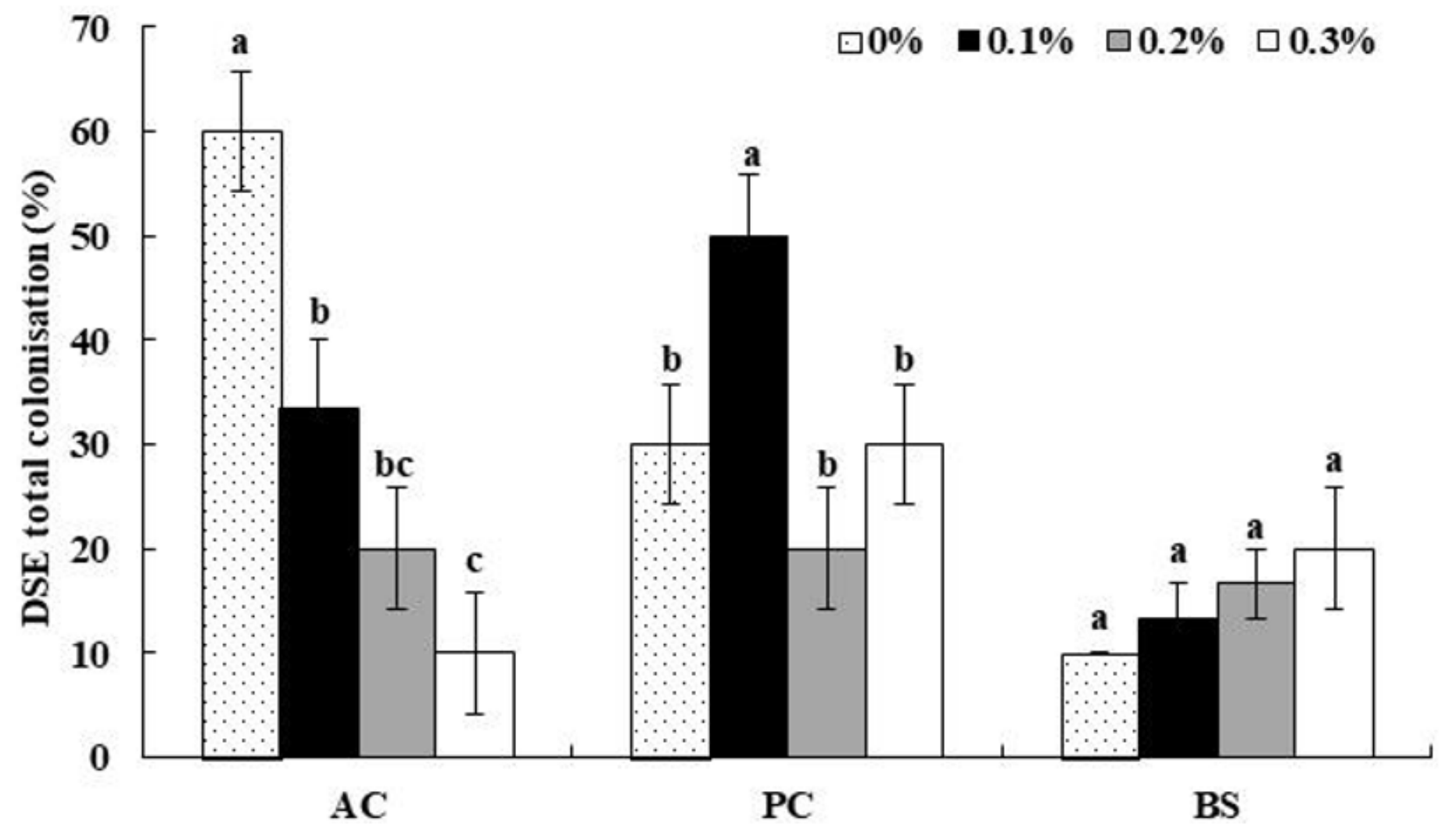

Figure 2

Total colonization rate of dark septate endophytes (DSE) in the roots of inoculated Artemisia ordosica under $\mathrm{NaCl}$ stress. The error bars represent the standard errors (SE) of the means. The different letters above the error bars indicate significant differences at $\mathrm{P}<0.05$ according to Duncan's multiple-range test. ' $A C$ ' indicates plants inoculated with Alternaria chlamydosporigena. ' $P C$ ' indicates plants inoculated with Paraphoma chrysanthemicola. 'BS' indicates plants inoculated with Bipolaris sorokiniana. 


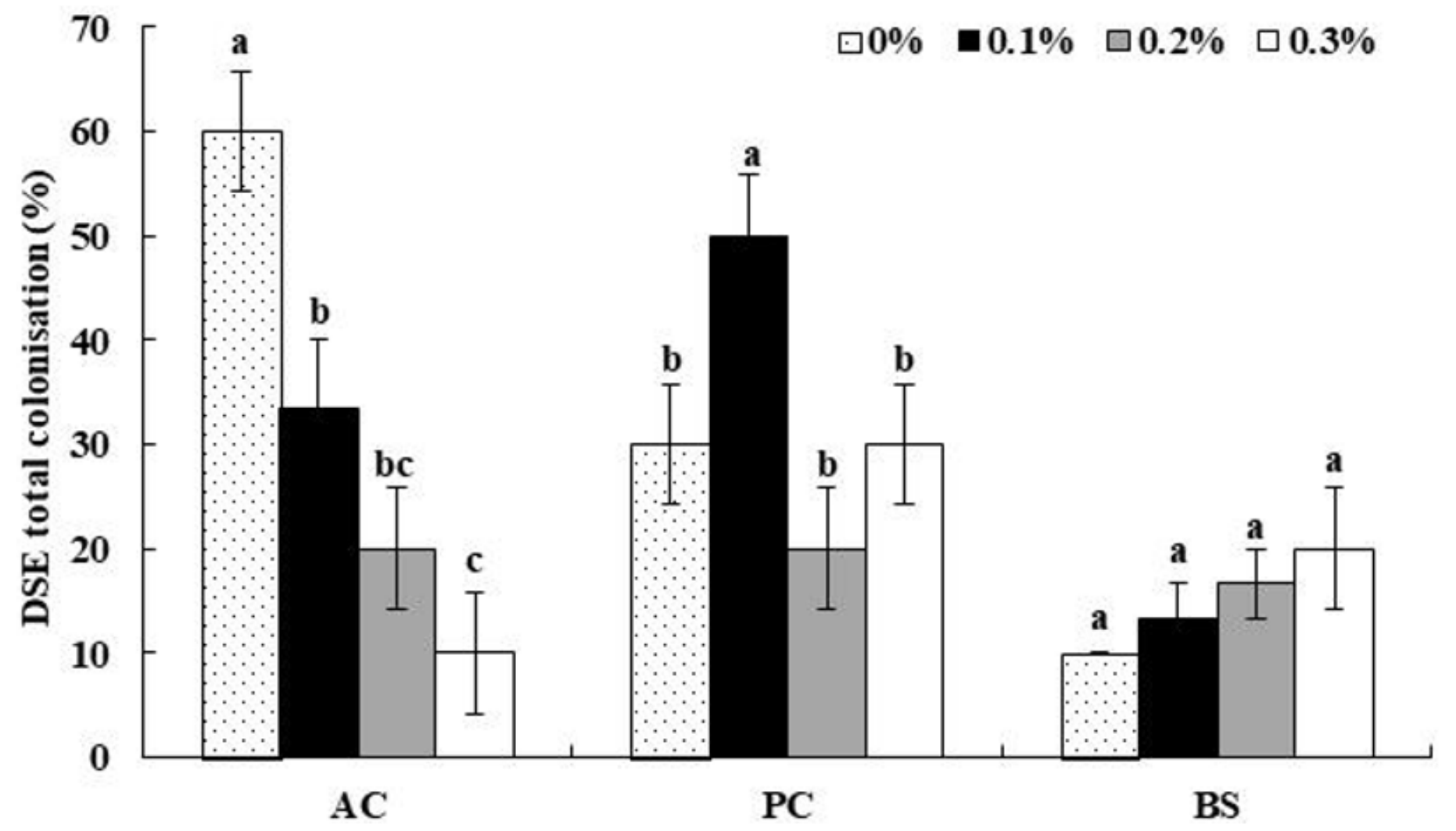

Figure 2

Total colonization rate of dark septate endophytes (DSE) in the roots of inoculated Artemisia ordosica under $\mathrm{NaCl}$ stress. The error bars represent the standard errors (SE) of the means. The different letters above the error bars indicate significant differences at $\mathrm{P}<0.05$ according to Duncan's multiple-range test. ' $A C$ ' indicates plants inoculated with Alternaria chlamydosporigena. ' $P C$ ' indicates plants inoculated with Paraphoma chrysanthemicola. 'BS' indicates plants inoculated with Bipolaris sorokiniana. 


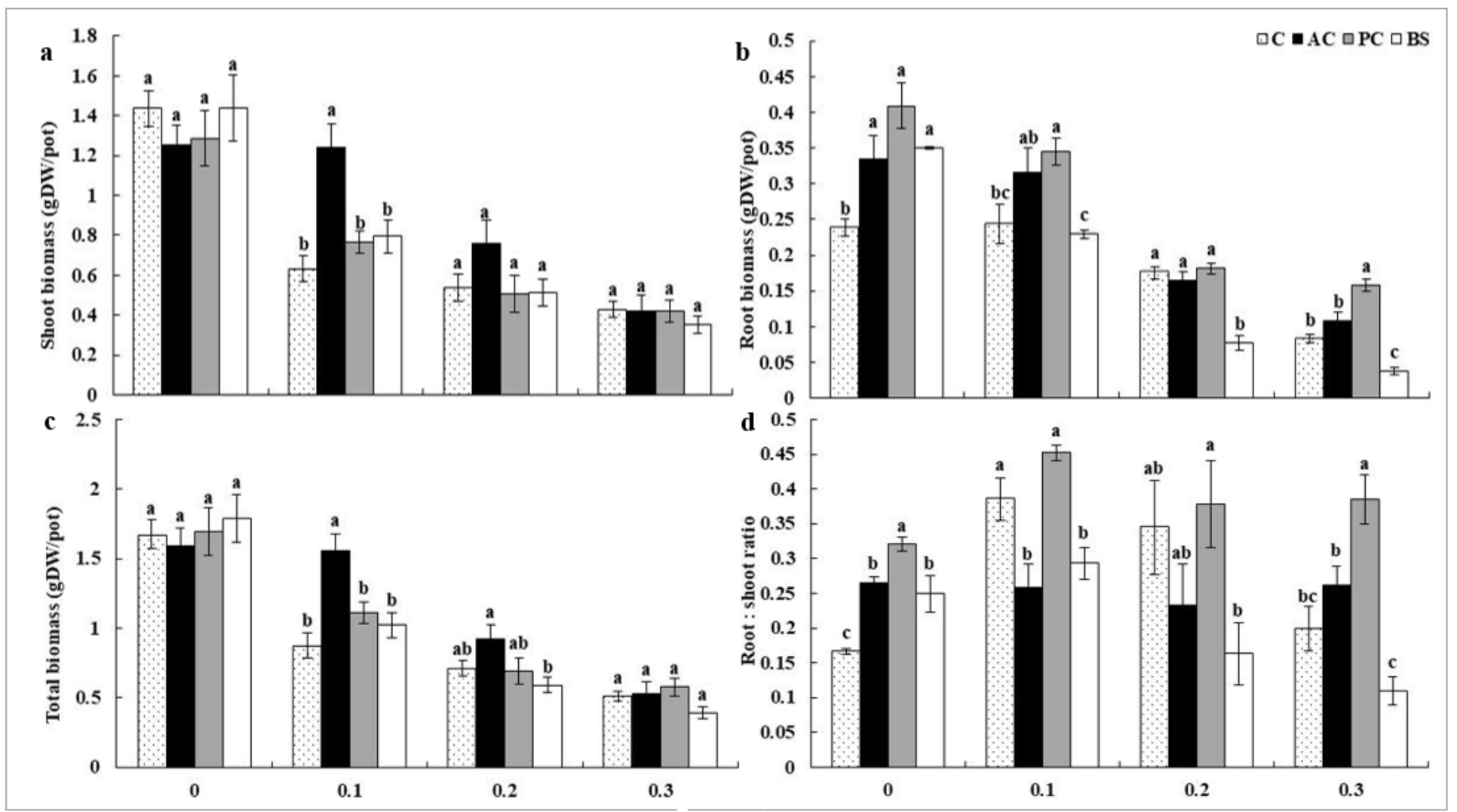

Figure 3

The effects of dark septate endophytes (DSE) inoculation and $\mathrm{NaCl}$ stress on the biomass production and root: shoot ratio of Artemisia ordosica. The error bars represent the standard errors (SE) of the means. The different letters above the error bars indicate significant differences at $\mathrm{P}<0.05$ according to Duncan's multiple-range test. ' $C$ ' indicates noninoculated plants. 'AC' indicates plants inoculated with Alternaria chlamydosporigena. 'PC' indicates plants inoculated with Paraphoma chrysanthemicola. 'BS' indicates plants inoculated with Bipolaris sorokiniana. DW indicates dry weight. 


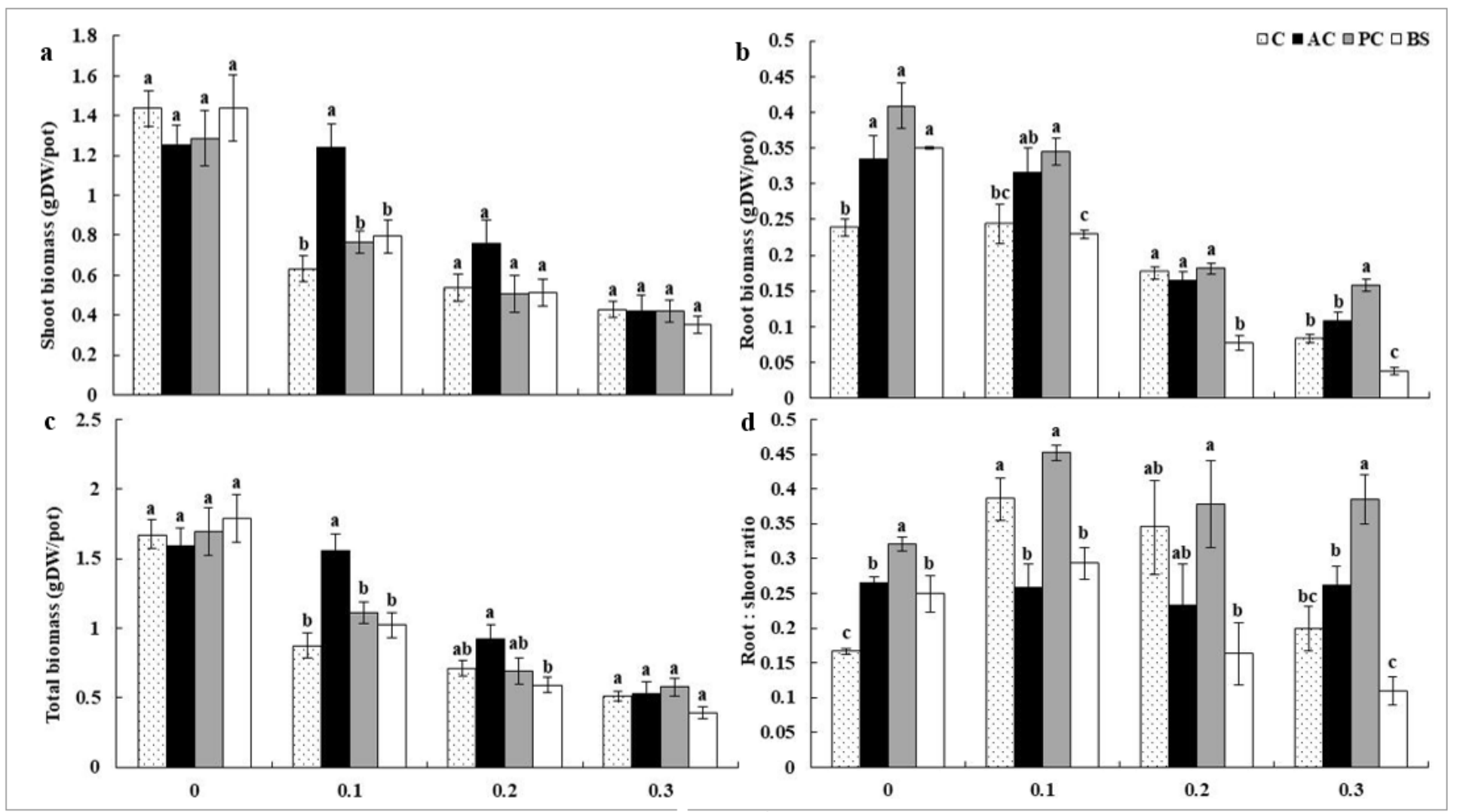

Figure 3

The effects of dark septate endophytes (DSE) inoculation and $\mathrm{NaCl}$ stress on the biomass production and root: shoot ratio of Artemisia ordosica. The error bars represent the standard errors (SE) of the means. The different letters above the error bars indicate significant differences at $\mathrm{P}<0.05$ according to Duncan's multiple-range test. ' $C$ ' indicates noninoculated plants. 'AC' indicates plants inoculated with Alternaria chlamydosporigena. 'PC' indicates plants inoculated with Paraphoma chrysanthemicola. 'BS' indicates plants inoculated with Bipolaris sorokiniana. DW indicates dry weight. 


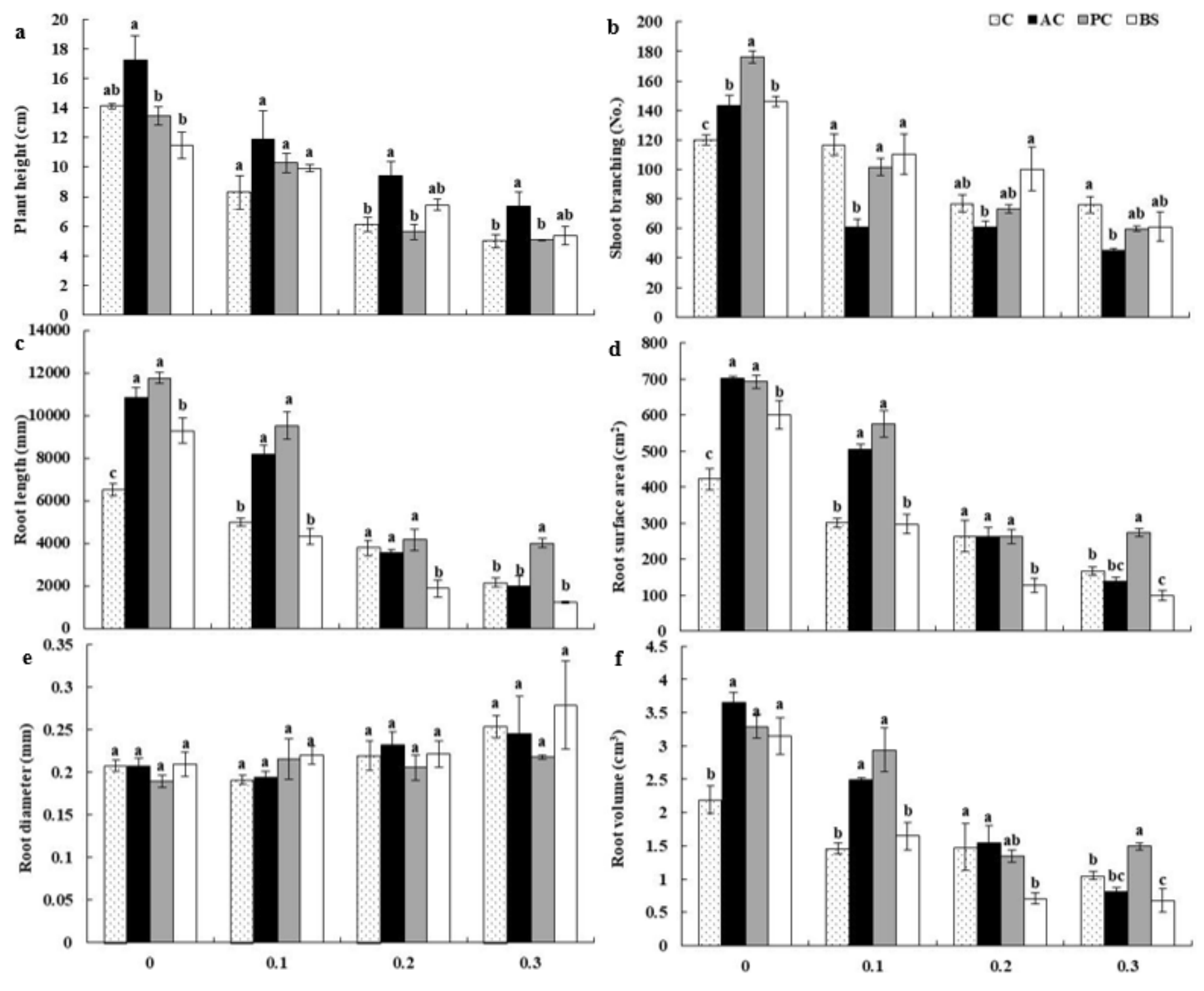

Figure 4

The effects of dark septate endophytes (DSE) inoculation and $\mathrm{NaCl}$ stress on the morphology of Artemisia ordosica. The error bars represent the standard errors (SE) of the means. The different letters above the error bars indicate significant differences at $\mathrm{P}<0.05$ according to Duncan's multiple-range test. ' $C$ ' indicates noninoculated plants. 'AC' indicates plants inoculated with Alternaria chlamydosporigena. 'PC' indicates plants inoculated with Paraphoma chrysanthemicola. 'BS' indicates plants inoculated with Bipolaris sorokiniana. 


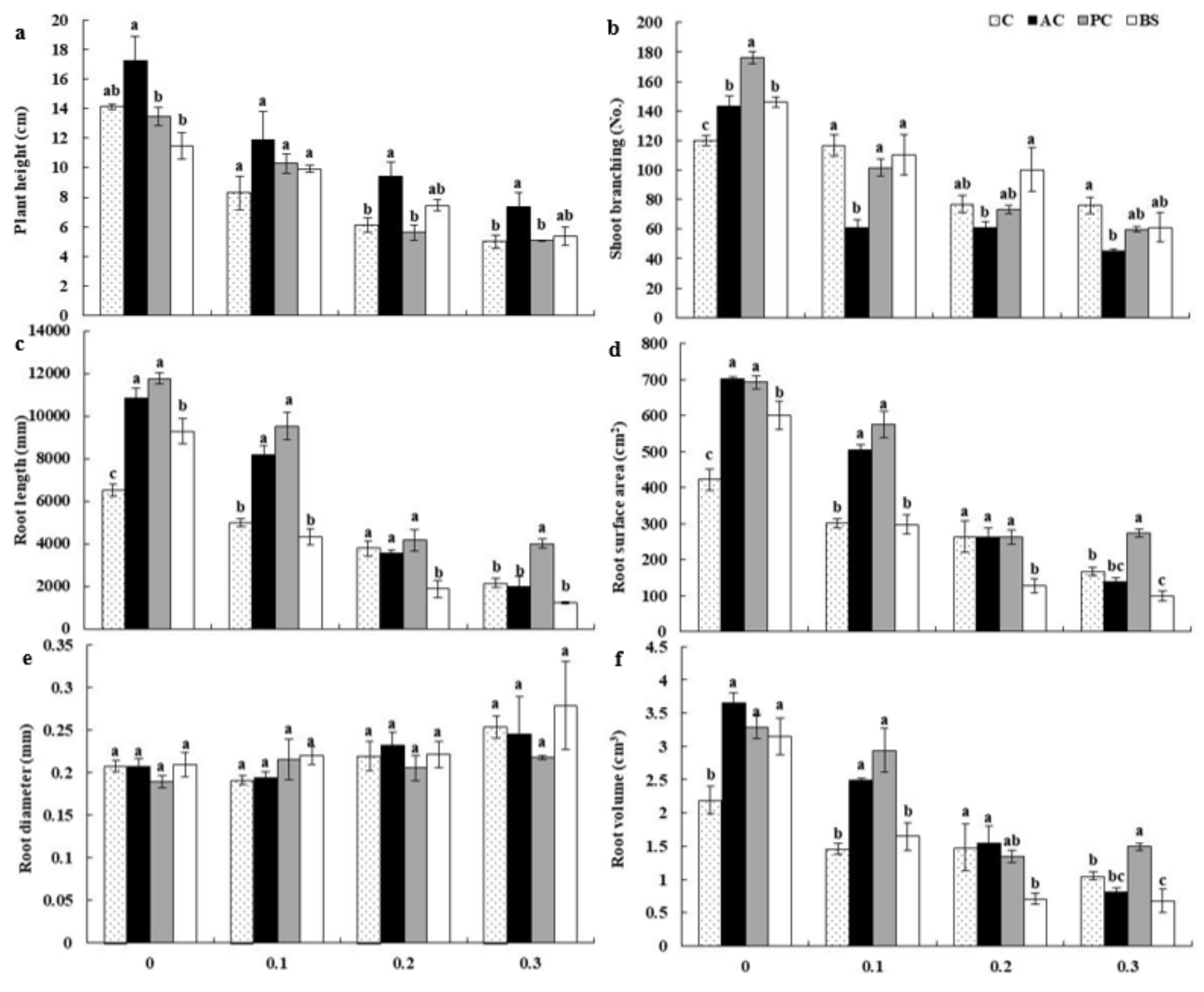

Figure 4

The effects of dark septate endophytes (DSE) inoculation and $\mathrm{NaCl}$ stress on the morphology of Artemisia ordosica. The error bars represent the standard errors (SE) of the means. The different letters above the error bars indicate significant differences at $\mathrm{P}<0.05$ according to Duncan's multiple-range test. ' $C$ ' indicates noninoculated plants. 'AC' indicates plants inoculated with Alternaria chlamydosporigena. 'PC' indicates plants inoculated with Paraphoma chrysanthemicola. 'BS' indicates plants inoculated with Bipolaris sorokiniana. 

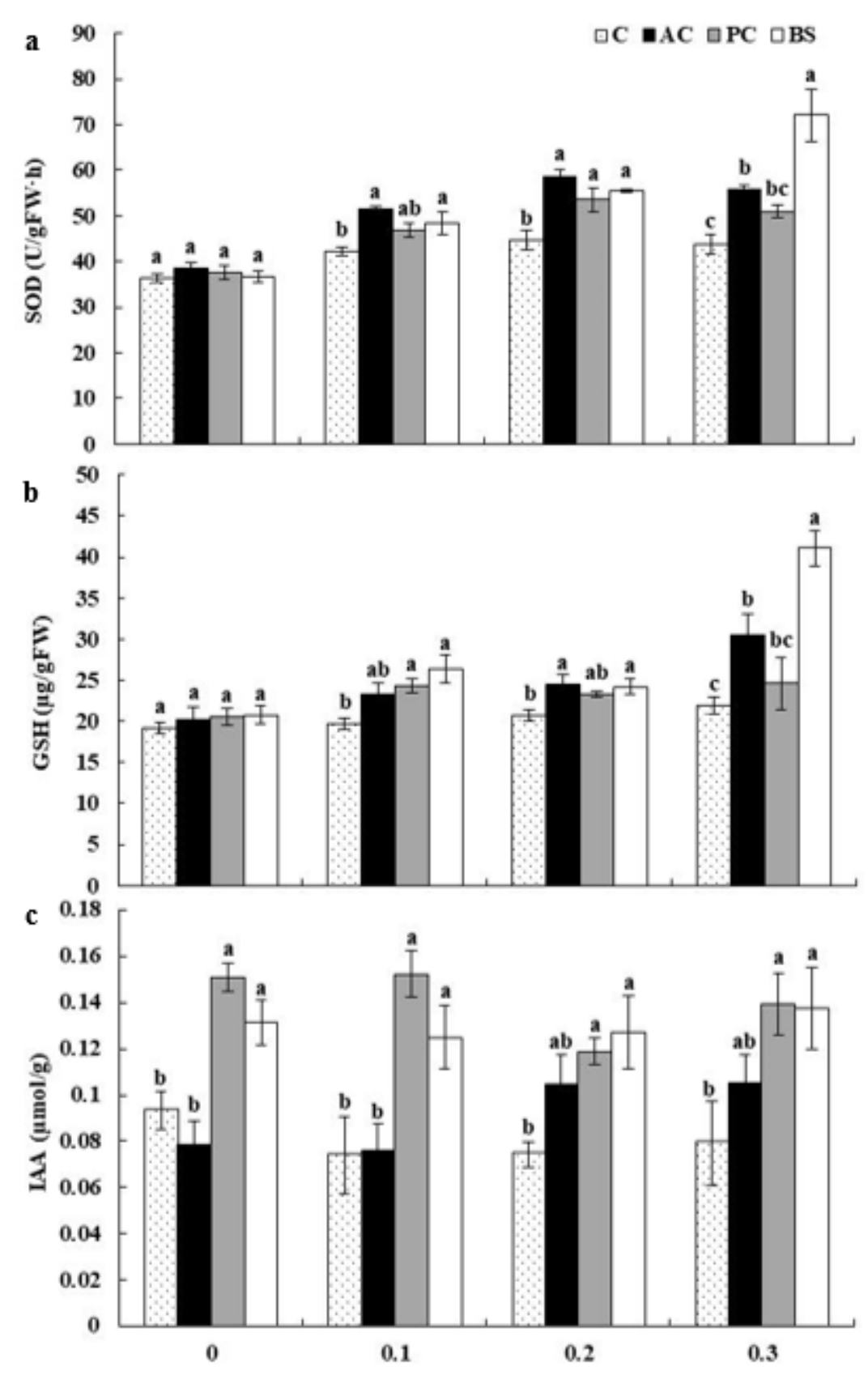

Figure 5

The effects of dark septate endophytes (DSE) inoculation and $\mathrm{NaCl}$ stress on the morphology of Artemisia ordosica. The error bars represent the standard errors (SE) of the means. The different letters above the error bars indicate significant differences at $P<0.05$ according to Duncan's multiple-range test. ' $C$ ' indicates noninoculated plants. 'AC' indicates plants inoculated with Alternaria chlamydosporigena. 'PC' indicates plants inoculated with Paraphoma chrysanthemicola. 'BS' indicates plants inoculated with Bipolaris sorokiniana. 

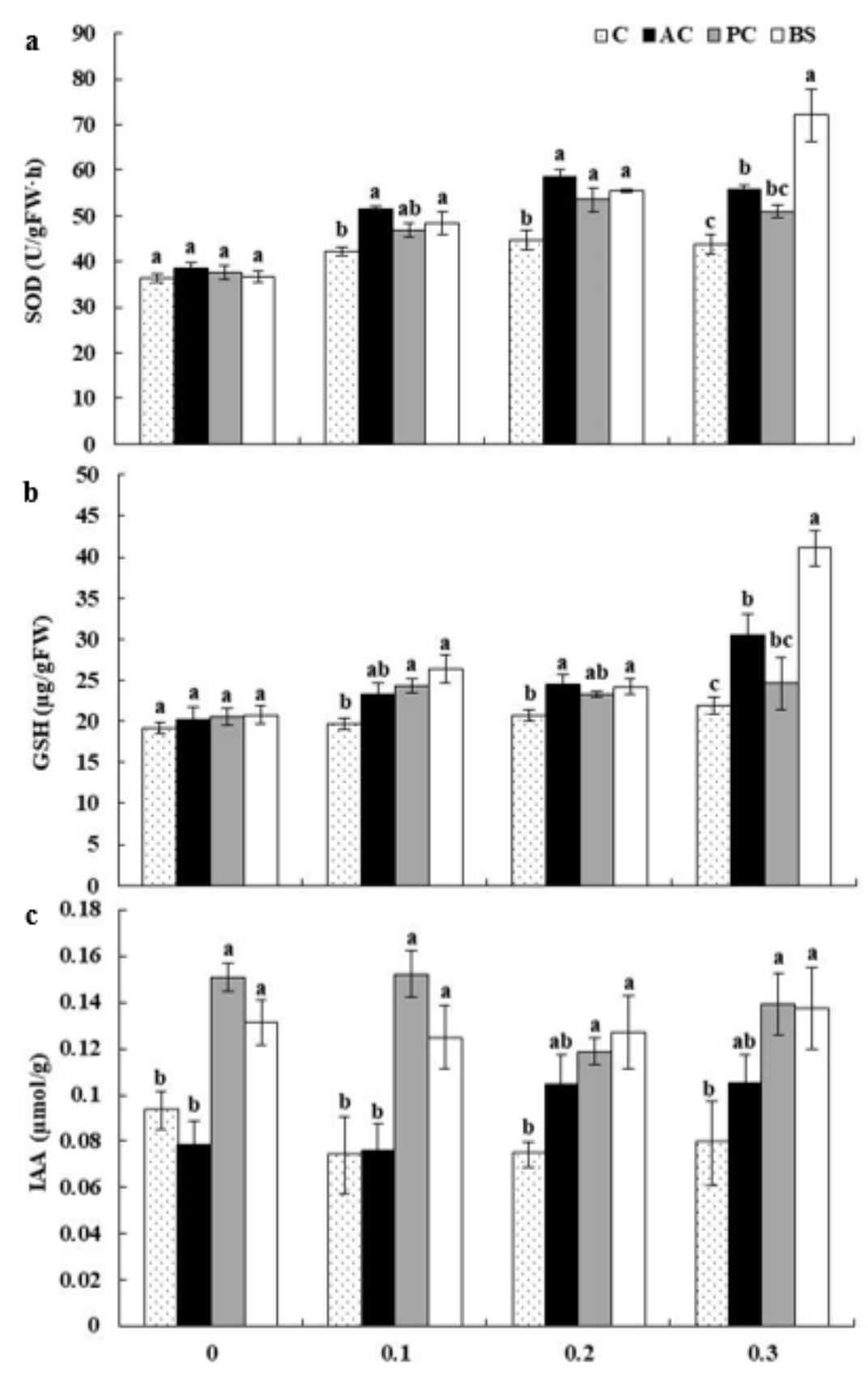

Figure 5

The effects of dark septate endophytes (DSE) inoculation and $\mathrm{NaCl}$ stress on the morphology of Artemisia ordosica. The error bars represent the standard errors (SE) of the means. The different letters above the error bars indicate significant differences at $P<0.05$ according to Duncan's multiple-range test. ' $C$ ' indicates noninoculated plants. 'AC' indicates plants inoculated with Alternaria chlamydosporigena. 'PC' indicates plants inoculated with Paraphoma chrysanthemicola. 'BS' indicates plants inoculated with Bipolaris sorokiniana. 


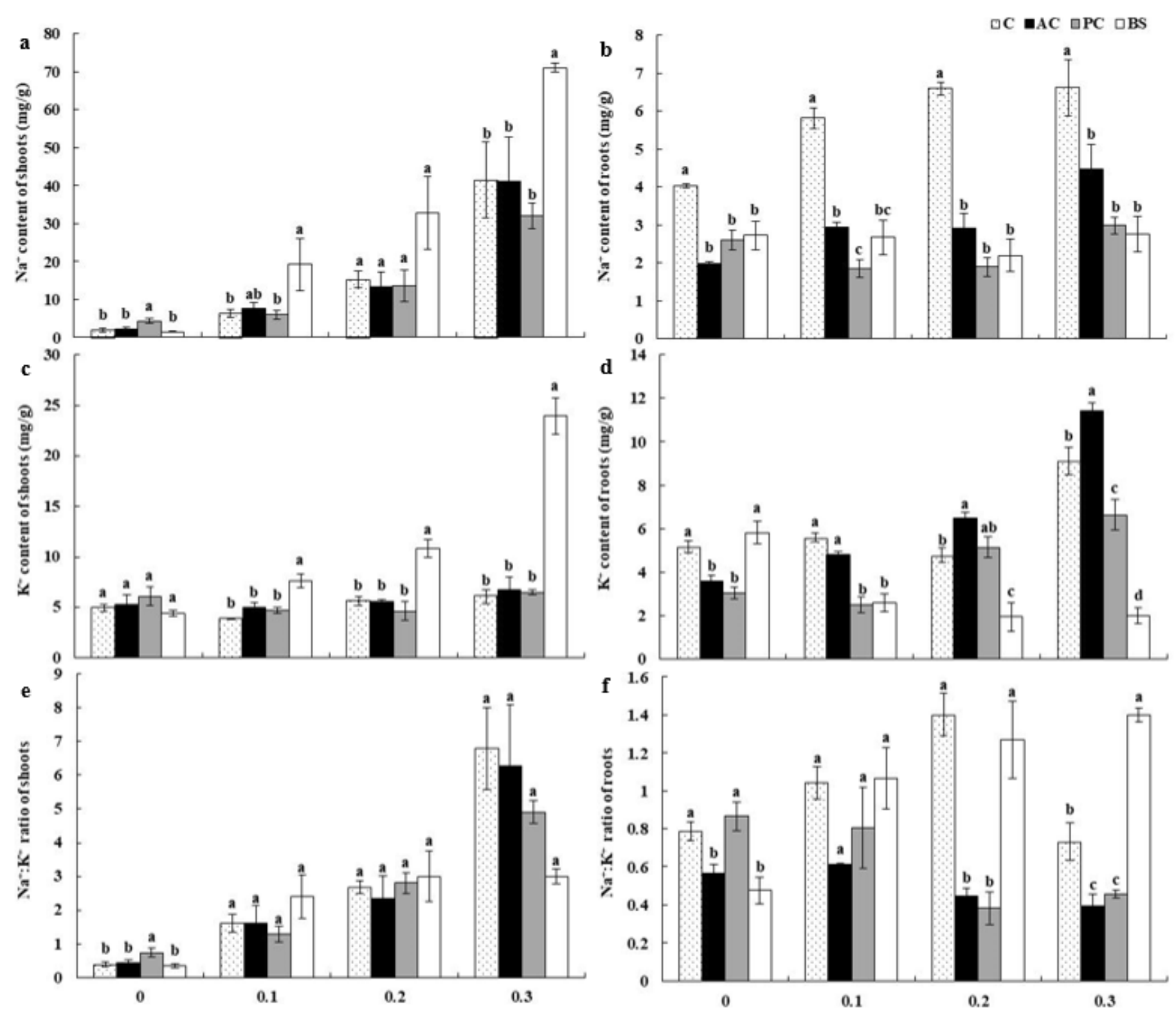

Figure 6

The effects of dark septate endophytes (DSE) inoculation and $\mathrm{NaCl}$ stress on the $\mathrm{Na}+$ and $\mathrm{K}+$ contents and $\mathrm{Na}+\mathrm{K}+$ ratio of Artemisia ordosica. The error bars represent the standard errors (SE) of the means. The different letters above the error bars indicate significant differences at $\mathrm{P}<0.05$ according to Duncan's multiple-range test. ' $C$ ' indicates noninoculated plants. 'AC' indicates plants inoculated with Alternaria chlamydosporigena. 'PC' indicates plants inoculated with Paraphoma chrysanthemicola. 'BS' indicates plants inoculated with Bipolaris sorokiniana. 


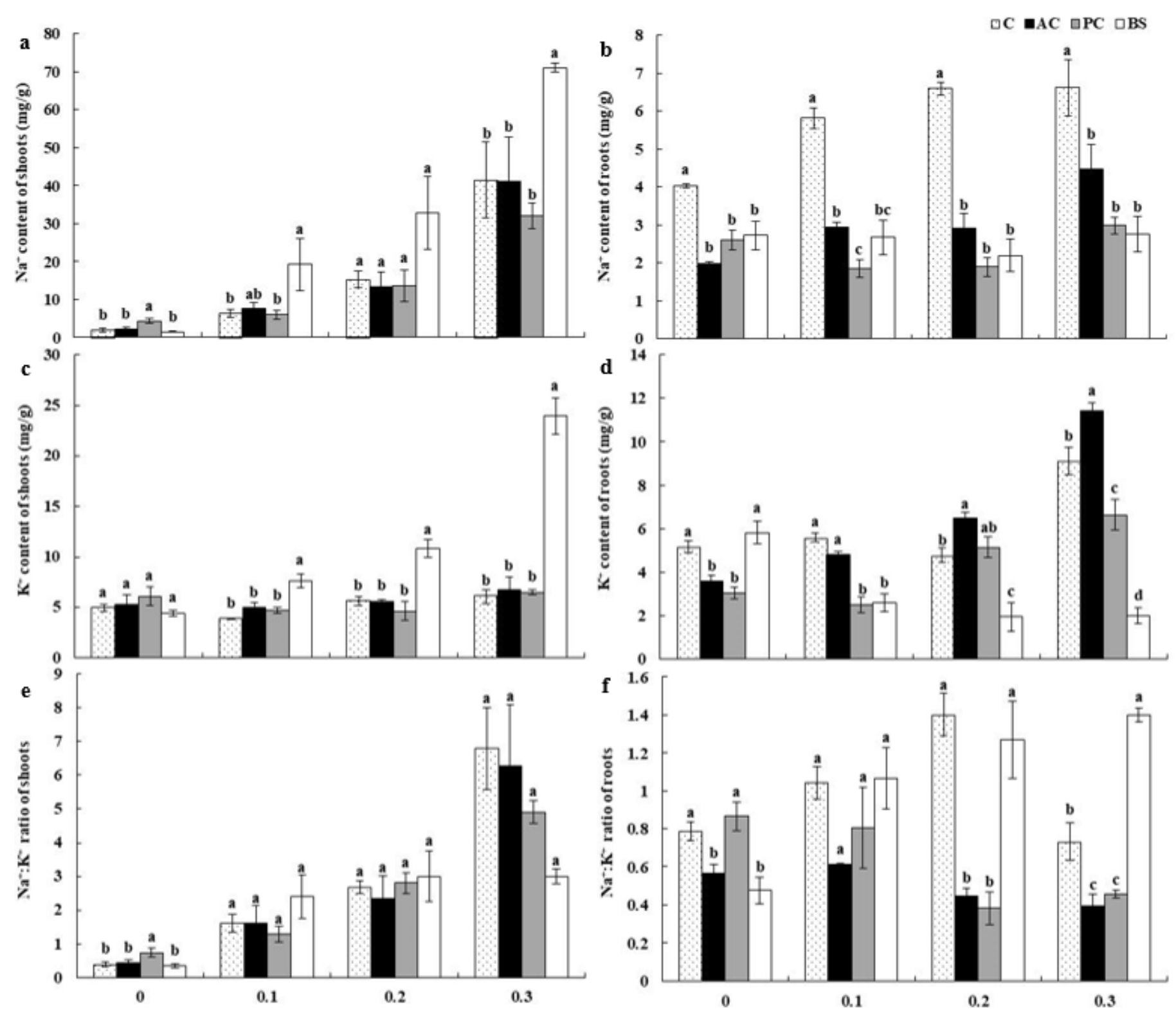

Figure 6

The effects of dark septate endophytes (DSE) inoculation and $\mathrm{NaCl}$ stress on the $\mathrm{Na}+$ and $\mathrm{K}+$ contents and $\mathrm{Na}+\mathrm{K}+$ ratio of Artemisia ordosica. The error bars represent the standard errors (SE) of the means. The different letters above the error bars indicate significant differences at $\mathrm{P}<0.05$ according to Duncan's multiple-range test. ' $C$ ' indicates noninoculated plants. 'AC' indicates plants inoculated with Alternaria chlamydosporigena. 'PC' indicates plants inoculated with Paraphoma chrysanthemicola. 'BS' indicates plants inoculated with Bipolaris sorokiniana. 

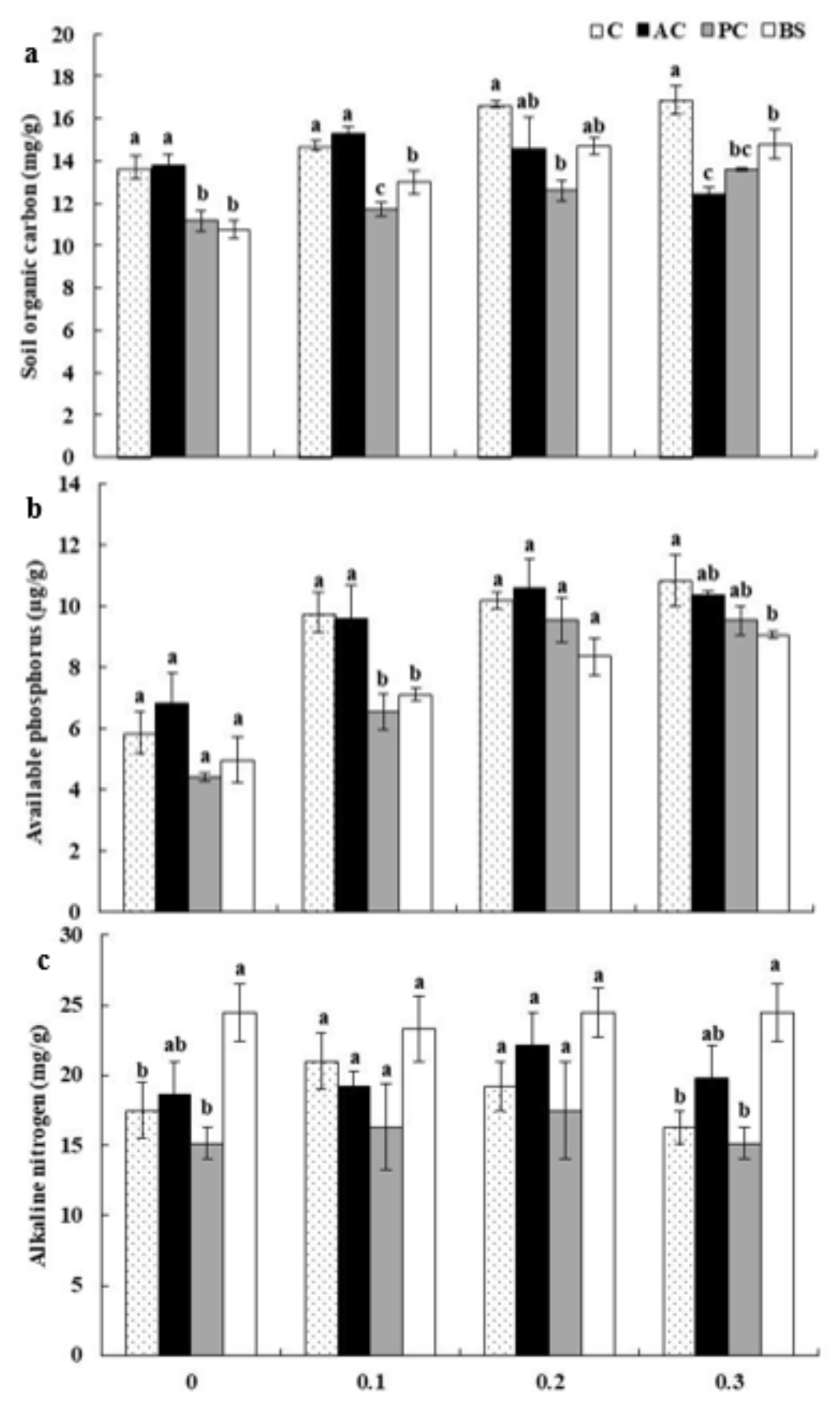

Figure 7

Effects of dark septate endophytes (DSE) inoculation and $\mathrm{NaCl}$ stress on the soil physicochemical properties in rhizosphere of Artemisia ordosica. The error bars represent the standard errors (SE) of the means. The different letters above the error bars indicate significant differences at $P<0.05$ according to Duncan's multiple-range test. ' $C$ ' indicates noninoculated plants. 'AC' indicates plants inoculated with Alternaria chlamydosporigena. 'PC' indicates plants inoculated with Paraphoma chrysanthemicola. 'BS' indicates plants inoculated with Bipolaris sorokiniana. 

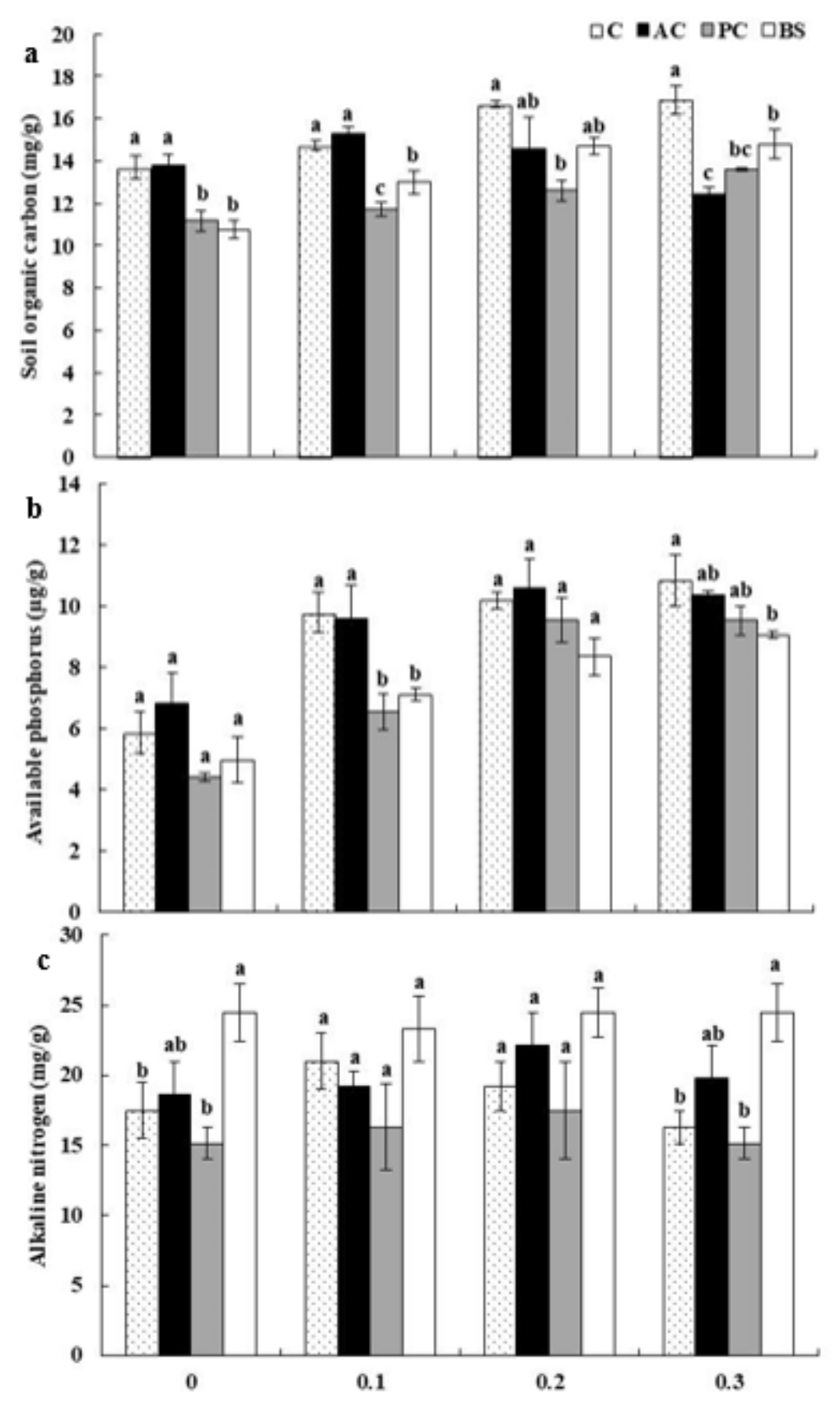

Figure 7

Effects of dark septate endophytes (DSE) inoculation and $\mathrm{NaCl}$ stress on the soil physicochemical properties in rhizosphere of Artemisia ordosica. The error bars represent the standard errors (SE) of the means. The different letters above the error bars indicate significant differences at $P<0.05$ according to Duncan's multiple-range test. ' $C$ ' indicates noninoculated plants. 'AC' indicates plants inoculated with Alternaria chlamydosporigena. 'PC' indicates plants inoculated with Paraphoma chrysanthemicola. 'BS' indicates plants inoculated with Bipolaris sorokiniana. 

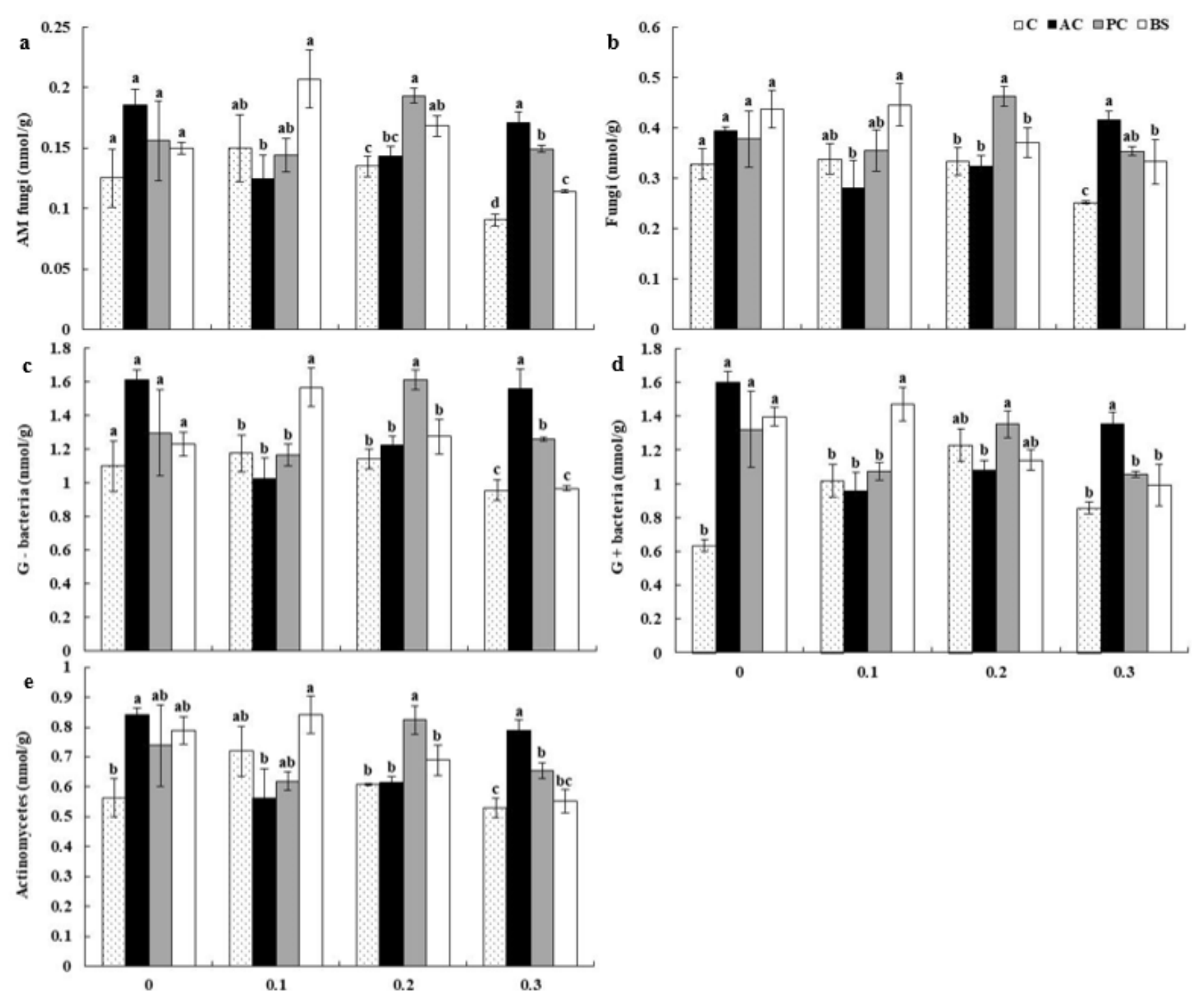

Figure 8

Effects of dark septate endophytes (DSE) inoculation and $\mathrm{NaCl}$ stress on the soil microbial community composition in the rhizosphere of Artemisia ordosica. The error bars represent the standard errors (SE) of the means. The different letters above the error bars indicate significant differences at $\mathrm{P}<0.05$ according to Duncan's multiple-range test. ' $C$ ' indicates noninoculated plants. ' $A C$ ' indicates plants inoculated with Alternaria chlamydosporigena. 'PC' indicates plants inoculated with Paraphoma chrysanthemicola. 'BS' indicates plants inoculated with Bipolaris sorokiniana. 

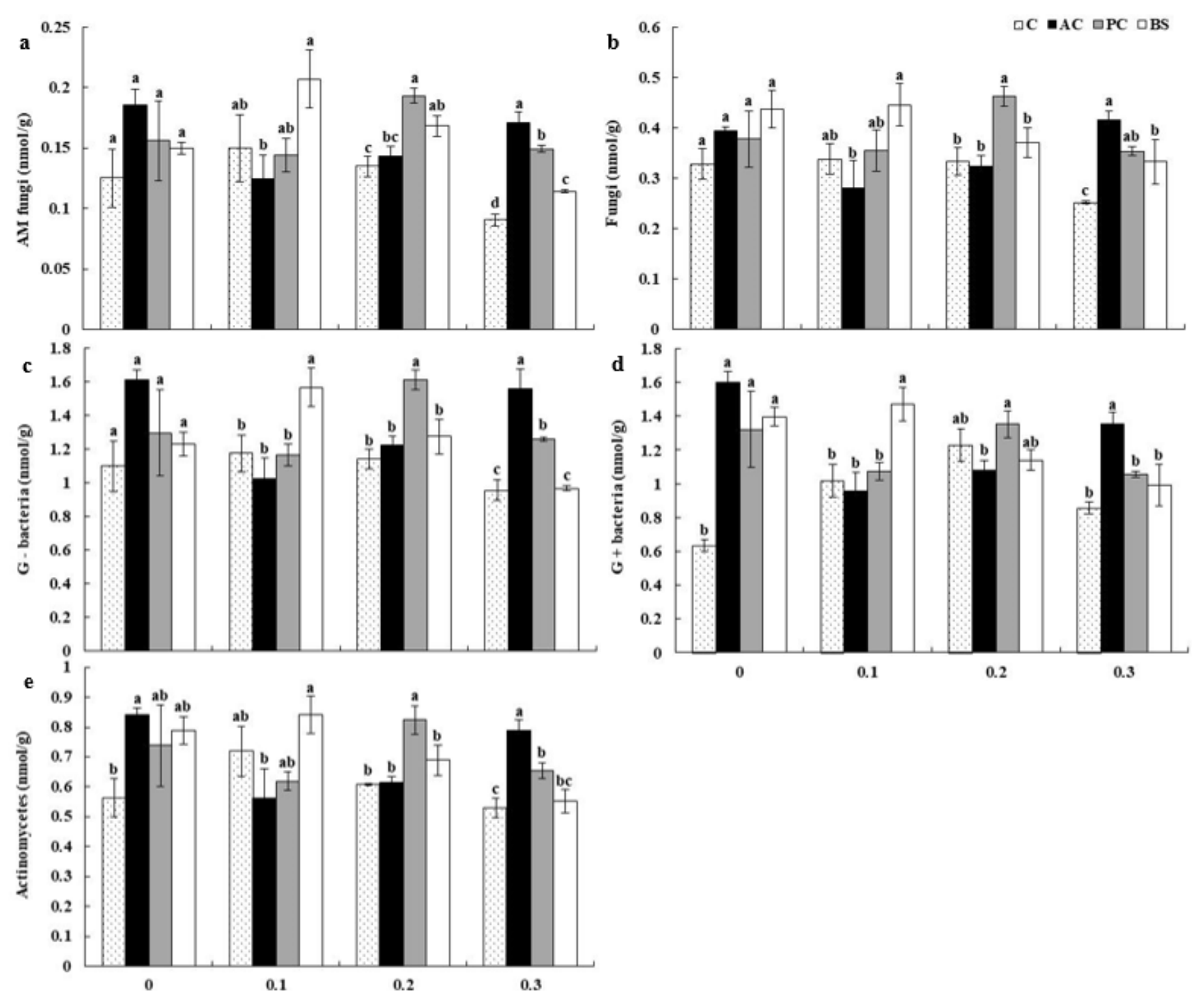

Figure 8

Effects of dark septate endophytes (DSE) inoculation and $\mathrm{NaCl}$ stress on the soil microbial community composition in the rhizosphere of Artemisia ordosica. The error bars represent the standard errors (SE) of the means. The different letters above the error bars indicate significant differences at $\mathrm{P}<0.05$ according to Duncan's multiple-range test. ' $C$ ' indicates noninoculated plants. ' $A C$ ' indicates plants inoculated with Alternaria chlamydosporigena. 'PC' indicates plants inoculated with Paraphoma chrysanthemicola. 'BS' indicates plants inoculated with Bipolaris sorokiniana. 


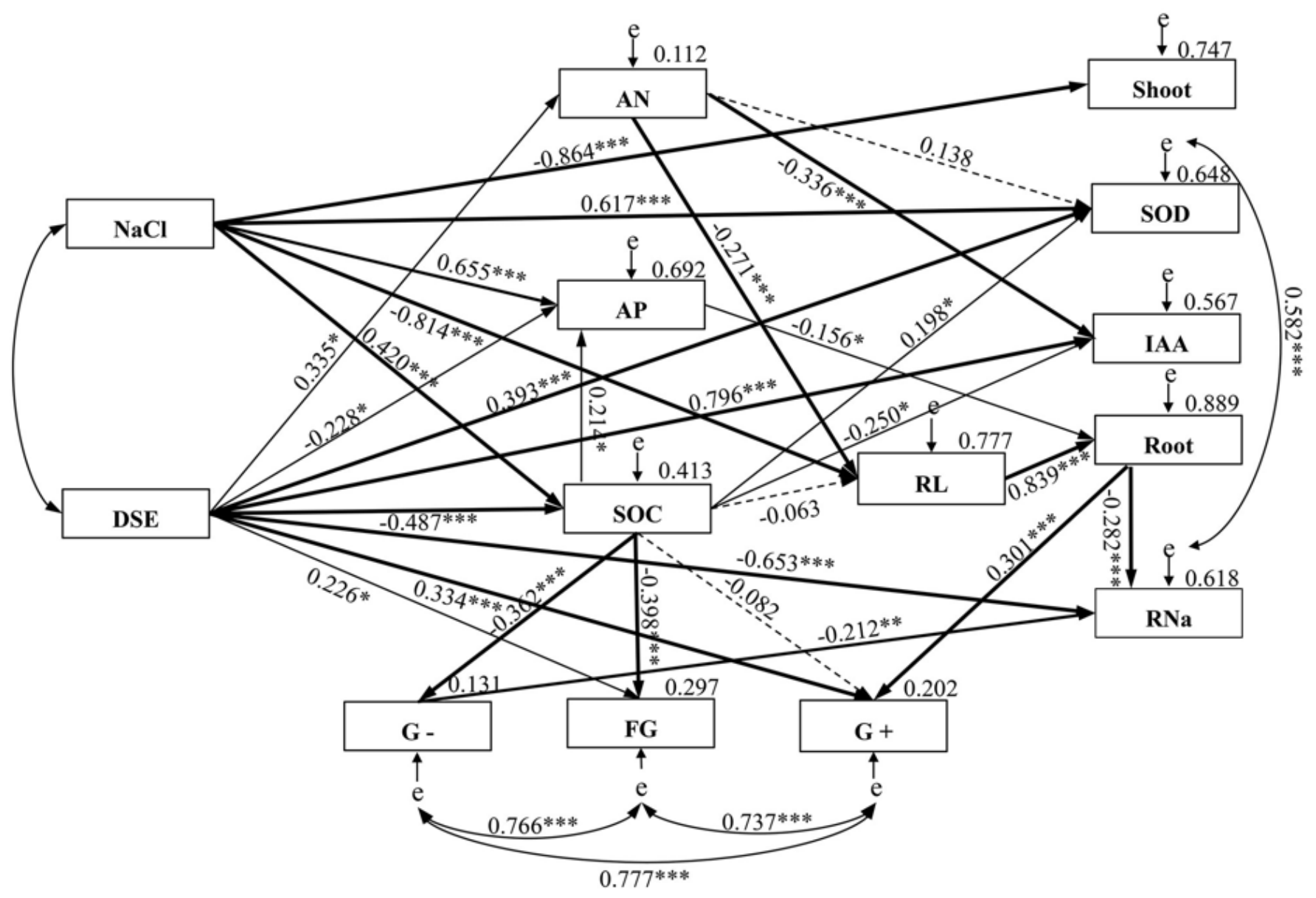

Figure 9

Structural equation model (SEM) showing the causal relationships among DSE species, $\mathrm{NaCl}$ stress, soil factors, the soil microbial community, and the growth parameters of Artemisia ordosica. The final model fit the data well: maximum likelihood, $\chi 2=78.527, \mathrm{df}=60, \mathrm{P}=0.055$, Tacker-Lewis index $(\mathrm{TLI})=0.953$, Comparative fit index $(\mathrm{CFI})=0.969$, and Root mean square error of approximation (RMSEA) $=0.081$. The solid lines and dashed lines indicate significant and nonsignificant pathways, respectively. The width of the solid lines indicates the strength of the causal effect, and the numbers near the arrows indicate the standardized path coefficients. Significant values are indicated by * $(P<0.05)$, ** $(P<0.01)$ and *** $(P<$ 0.001). The numbers in the upper-right corner of the box indicate the R2 values and represent the proportion of variance explained for each variable. $\mathrm{SOC}=$ soil organic carbon. $\mathrm{AP}=$ soil available phosphorus. $\mathrm{AN}=$ soil alkaline nitrogen. $\mathrm{G}$ - = gram-negative bacteria. $\mathrm{FG}=$ fungi. $\mathrm{G}+$ = gram-positive bacteria. Shoot $=$ shoot biomass. Root $=$ root biomass. SOD $=$ superoxide dismutase activity. IAA = indoleacetic acid content. $\mathrm{RL}=$ root length. $\mathrm{RNa}=\mathrm{Na}+$ content in the root. 


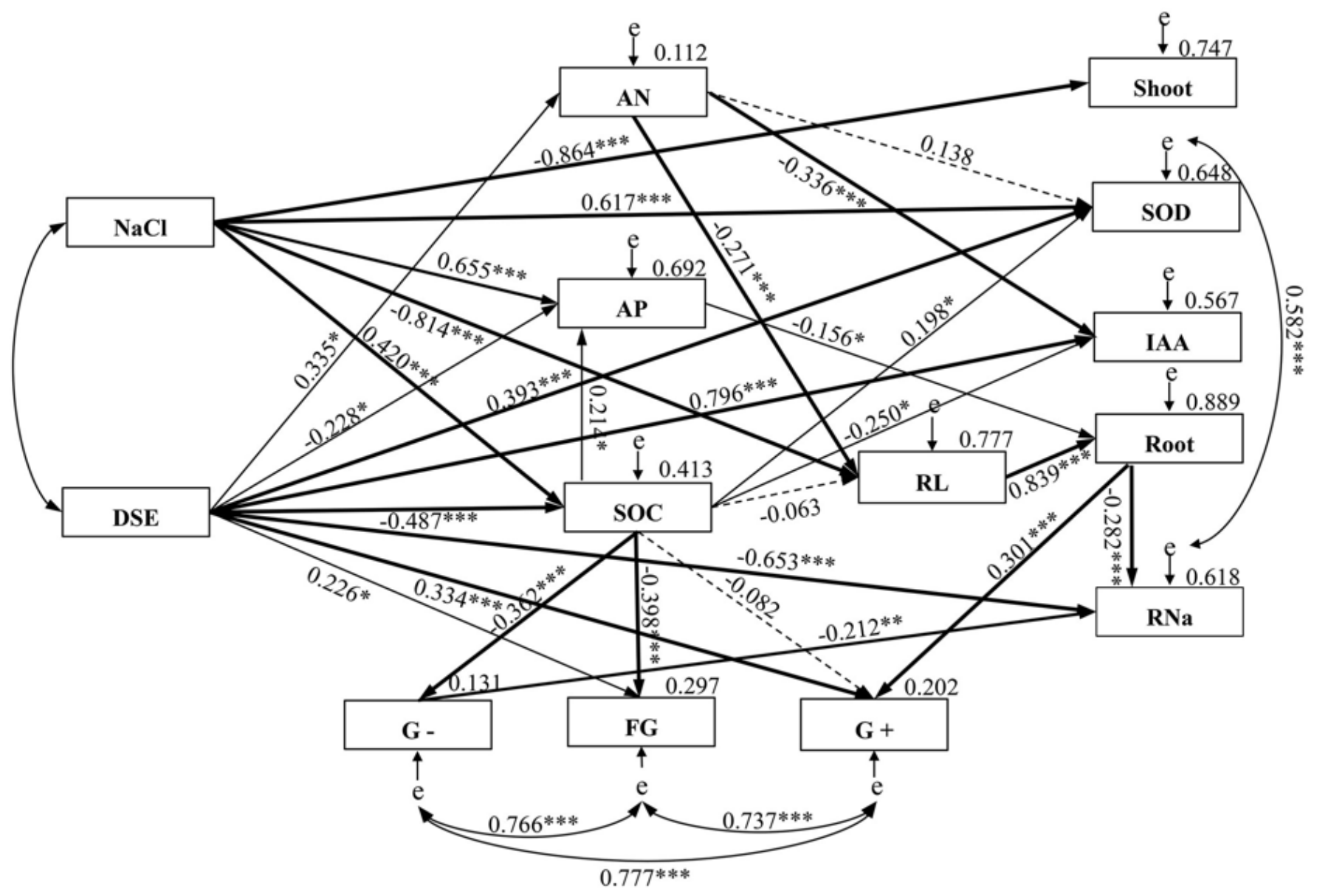

Figure 9

Structural equation model (SEM) showing the causal relationships among DSE species, $\mathrm{NaCl}$ stress, soil factors, the soil microbial community, and the growth parameters of Artemisia ordosica. The final model fit the data well: maximum likelihood, $\chi 2=78.527, \mathrm{df}=60, \mathrm{P}=0.055$, Tacker-Lewis index $(\mathrm{TLI})=0.953$, Comparative fit index $(\mathrm{CFI})=0.969$, and Root mean square error of approximation (RMSEA) $=0.081$. The solid lines and dashed lines indicate significant and nonsignificant pathways, respectively. The width of the solid lines indicates the strength of the causal effect, and the numbers near the arrows indicate the standardized path coefficients. Significant values are indicated by * $(P<0.05)$, ** $(P<0.01)$ and *** $(P<$ 0.001). The numbers in the upper-right corner of the box indicate the R2 values and represent the proportion of variance explained for each variable. $\mathrm{SOC}=$ soil organic carbon. $\mathrm{AP}=$ soil available phosphorus. $\mathrm{AN}=$ soil alkaline nitrogen. $\mathrm{G}-$ = gram-negative bacteria. $\mathrm{FG}=$ fungi. $\mathrm{G}+$ = gram-positive bacteria. Shoot $=$ shoot biomass. Root $=$ root biomass. SOD $=$ superoxide dismutase activity. IAA = indoleacetic acid content. $\mathrm{RL}=$ root length. $\mathrm{RNa}=\mathrm{Na}+$ content in the root.

\section{Supplementary Files}

This is a list of supplementary files associated with this preprint. Click to download. 
- SupplementarymaterialLifengHou.docx

- SupplementarymaterialLifengHou.docx 